Publ. Mat. 61 (2017), 363-394

DOI: 10.5565 /PUBLMAT6121703

\title{
IRREDUCIBLE REPRESENTATIONS OF KNOT GROUPS INTO $\operatorname{SL}(n, \mathrm{C})$
}

\author{
Leila Ben AbDelghani And Michael Heusener
}

\begin{abstract}
The aim of this article is to study the existence of certain reducible, metabelian representations of knot groups into $\mathrm{SL}(n, \mathbf{C})$ which generalize the representations studied previously by G. Burde and G. de Rham. Under specific hypotheses we prove the existence of irreducible deformations of such representations of knot groups into $\mathrm{SL}(n, \mathbf{C})$.
\end{abstract}

2010 Mathematics Subject Classification: 57M25, 57M05, 57M27.

Key words: Knot group, Alexander module, representation variety.

\section{Introduction}

In [3] the authors studied the deformations of certain metabelian, reducible representations of knot groups into $\mathrm{SL}(3, \mathbf{C})$. In this paper we continue this study by generalizing the results of $[\mathbf{3}]$ to the group $\mathrm{SL}(n, \mathbf{C})$ (see Theorem 1.1).

Let $\Gamma$ be a finitely generated group. The set $R_{n}(\Gamma):=R(\Gamma, \operatorname{SL}(n, \mathbf{C}))$ of homomorphisms of $\Gamma$ in $\mathrm{SL}(n, \mathbf{C})$ is called the $\mathrm{SL}(n, \mathbf{C})$-representation variety of $\Gamma$. It is a (not necessarily irreducible) algebraic variety. A representation $\rho: \Gamma \rightarrow \mathrm{SL}(n, \mathbf{C})$ is called abelian (resp. metabelian) if the restriction of $\rho$ to the first (resp. second) commutator subgroup of $\Gamma$ is trivial. The representation $\rho: \Gamma \rightarrow \mathrm{SL}(n)$ is called reducible if there exists a proper subspace $V \subset \mathbf{C}^{n}$ such that $\rho(\Gamma)$ preserves $V$. Otherwise $\rho$ is called irreducible.

Let $K \subset M^{3}$ be a knot in a three-dimensional integer homology sphere $M^{3}$. We let $\Gamma=\Gamma_{K}$ denote the knot group of $K$ i.e. $\Gamma_{K}$ is the fundamental group of the knot complement $M^{3} \backslash K$. Since the ring of complex Laurent polynomials $\mathbf{C}\left[t^{ \pm 1}\right]$ is a principal ideal domain, the complex Alexander module $A(t)$ of $K$ decomposes into a direct sum of cyclic modules. A generator of the order ideal of $A(t)$ is called the Alexander polynomial of $K$. It will be denoted by $\Delta_{K}(t) \in \mathbf{C}\left[t^{ \pm 1}\right]$, and it is unique up to multiplication by a unit $c t^{k} \in \mathbf{C}\left[t^{ \pm 1}\right], c \in \mathbf{C}^{*}, k \in \mathbf{Z}$. 
For a given root $\alpha \in \mathbf{C}^{*}$ of $\Delta_{K}(t)$ we let $\tau_{\alpha}$ denote the $(t-\alpha)$-torsion of the Alexander module. (For details see Section 2.)

The main result of this article is the following theorem which generalizes the results of $[\mathbf{3}]$ where the case $n=3$ was investigated. It also applies in the case $n=2$ which was studied in [1] and [14, Theorem 1.1].

Theorem 1.1. Let $K$ be a knot in the 3-dimensional integer homology sphere $M^{3}$. If the $(t-\alpha)$-torsion $\tau_{\alpha}$ of the Alexander module is cyclic of the form $\mathbf{C}\left[t^{ \pm 1}\right] /(t-\alpha)^{n-1}, n \geq 2$, then for each $\lambda \in \mathbf{C}^{*}$ such that $\lambda^{n}=\alpha$ there exists a certain reducible metabelian representation $\varrho_{\lambda}$ of the knot group $\Gamma$ into $\mathrm{SL}(n, \mathbf{C})$. Moreover, the representation $\varrho_{\lambda}$ is a smooth point of the representation variety $R_{n}(\Gamma)$. It is contained in a unique $\left(n^{2}+n-2\right)$-dimensional component $R_{\varrho_{\lambda}}$ of $R_{n}(\Gamma)$ which contains irreducible non-metabelian representations which deform $\varrho_{\lambda}$.

This paper is organised as follows. In Section 2 we introduce some notations and recall some facts which will be used in this article. In Section 3 we study the existence of certain reducible representations. These representations were previously studied in [16], and we treat the existence results from a more general point of view. Section 4 is devoted to the proof of Proposition 4.1, and it contains all necessary cohomological calculations. In the last section we prove that there are irreducible non-metabelian deformations of the initial reducible representation.

Acknowledgements. Both authors are pleased to acknowledge the support by the French-Tunisian CMCU project $\mathrm{n}^{\circ} 12 \mathrm{G} / 1502$. Moreover, the first author was supported by the project Erasmus mundus E-GOVTN. The second author acknowledges support from the ANR projects SGT and ModGroup. Also, the authors like to thank the anonymous referee for her/his valuable remarks. Her/his suggestions helped to improve in a substantial way the exposition and the content of the paper.

\section{Notations and facts}

To shorten notation we will simply write $\operatorname{SL}(n)$ and $\operatorname{GL}(n)$ instead of $\operatorname{SL}(n, \mathbf{C})$ and $\operatorname{GL}(n, \mathbf{C})$ respectively. The same notation applies for the Lie algebras $\mathfrak{s l}(n)=\mathfrak{s l}(n, \mathbf{C})$ and $\mathfrak{g l}(n)=\mathfrak{g l}(n, \mathbf{C})$.

2.1. Group cohomology. The general reference for group cohomology is K. S. Brown's book [6]. Let $A$ be a $\Gamma$-module. We denote by $C^{*}(\Gamma ; A)$ the cochain complex; the coboundary operator $\delta: C^{n}(\Gamma ; A) \rightarrow C^{n+1}(\Gamma ; A)$ 
is given by:

$$
\begin{aligned}
\delta f\left(\gamma_{1}, \ldots, \gamma_{n+1}\right)= & \gamma_{1} \cdot f\left(\gamma_{2}, \ldots, \gamma_{n+1}\right) \\
& +\sum_{i=1}^{n}(-1)^{i} f\left(\gamma_{1}, \ldots, \gamma_{i-1}, \gamma_{i} \gamma_{i+1}, \ldots, \gamma_{n+1}\right) \\
& +(-1)^{n+1} f\left(\gamma_{1}, \ldots, \gamma_{n}\right) .
\end{aligned}
$$

The coboundaries (respectively cocycles, cohomology) of $\Gamma$ with coefficients in $A$ are denoted by $B^{*}(\Gamma ; A)$ (respectively $\left.Z^{*}(\Gamma ; A), H^{*}(\Gamma ; A)\right)$. In what follows 1-cocycles and 1-coboundaries will be also called derivations and principal derivations respectively.

Let $A_{1}, A_{2}$, and $A_{3}$ be $\Gamma$-modules. The cup product of two cochains $u \in C^{p}\left(\Gamma ; A_{1}\right)$ and $v \in C^{q}\left(\Gamma ; A_{2}\right)$ is the cochain $u \smile v \in C^{p+q}\left(\Gamma ; A_{1} \otimes A_{2}\right)$ defined by

(1) $u \smile v\left(\gamma_{1}, \ldots, \gamma_{p+q}\right):=u\left(\gamma_{1}, \ldots, \gamma_{p}\right) \otimes\left(\gamma_{1} \ldots \gamma_{p}\right) \cdot v\left(\gamma_{p+1}, \ldots, \gamma_{p+q}\right)$.

Here $A_{1} \otimes A_{2}$ is a $\Gamma$-module via the diagonal action. It is possible to combine the cup product with any $\Gamma$-invariant bilinear map $A_{1} \otimes A_{2} \rightarrow$ $A_{3}$. We are mainly interested in the product map $\mathbf{C} \otimes \mathbf{C} \rightarrow \mathbf{C}$.

Remark 2.1. Notice that our definition of the cup product (1) differs from the convention used in $\left[6\right.$, V.3] by the $\operatorname{sign}(-1)^{p q}$. Hence with the definition (1) the following formula holds:

$$
\delta(u \smile v)=(-1)^{q} \delta u \smile v+u \smile \delta v .
$$

A short exact sequence

$$
0 \longrightarrow A_{1} \stackrel{i}{\longrightarrow} A_{2} \stackrel{p}{\longrightarrow} A_{3} \longrightarrow 0
$$

of $\Gamma$-modules gives rise to a short exact sequence of cochain complexes:

$$
0 \longrightarrow C^{*}\left(\Gamma ; A_{1}\right) \stackrel{i^{*}}{\longrightarrow} C^{*}\left(\Gamma ; A_{2}\right) \stackrel{p^{*}}{\longrightarrow} C^{*}\left(\Gamma ; A_{3}\right) \longrightarrow 0 .
$$

We will make use of the corresponding long exact cohomology sequence (see [6, III. Proposition 6.1]):

$$
0 \longrightarrow H^{0}\left(\Gamma ; A_{1}\right) \longrightarrow H^{0}\left(\Gamma ; A_{2}\right) \longrightarrow H^{0}\left(\Gamma ; A_{3}\right) \stackrel{\beta^{0}}{\longrightarrow} H^{1}\left(\Gamma ; A_{1}\right) \longrightarrow \cdots
$$

Recall that the Bockstein homomorphism $\beta^{n}: H^{n}\left(\Gamma ; A_{3}\right) \rightarrow H^{n+1}\left(\Gamma ; A_{1}\right)$ is determined by the snake lemma: if $z \in Z^{n}\left(\Gamma ; A_{3}\right)$ is a cocycle and if $\tilde{z} \in\left(p^{*}\right)^{-1}(z) \subset C^{n}\left(\Gamma ; A_{2}\right)$ is any lift of $z$ then $\delta_{2}(\tilde{z}) \in \operatorname{Im}\left(i^{*}\right)$, where $\delta_{2}$ denotes the coboundary operator of $C^{*}\left(\Gamma ; A_{2}\right)$. It follows that any cochain $z^{\prime} \in C^{n+1}\left(\Gamma ; A_{3}\right)$ such that $i^{*}\left(z^{\prime}\right)=\delta_{2}(\tilde{z})$ is a cocycle and that its cohomology class only depends on the cohomology class represented 
by $z$. The cocycle $z^{\prime}$ represents the image of the cohomology class represented by $z$ under $\beta^{n}$.

Remark 2.2. By abuse of notation and if no confusion can arise, we will write sometimes $\beta^{n}(z)$ for a cocycle $z \in Z^{n}\left(\Gamma ; A_{3}\right)$ even if the map $\beta^{n}$ is only well defined on cohomology classes. This will simplify the notations.

We will make use of the following known fact [13, Lemma 3.1]:

Lemma 2.3. Let $\Gamma$ be a finitely presented group, and $A$ a $\Gamma$-module. Suppose that $X$ is any $C W$-complex with $\pi_{1}(X) \cong \Gamma$. Then there are natural morphisms $H_{i}(X ; A) \rightarrow H_{i}(\Gamma ; A)$ which are isomorphisms for $i=0,1$ and a surjection for $i=2$. In cohomology there are natural morphisms $H^{i}(\Gamma ; A) \rightarrow H^{i}(X ; A)$ which are isomorphisms for $i=0,1$ and an injection for $i=2$.

2.2. The Alexander module. Let $K \subset M^{3}$ be a knot in a threedimensional integer homology sphere $M^{3}$. We let $X=\overline{M^{3} \backslash V(K)}$ denote its complement where $V(K)$ is a tubular neighborhood of $K$. Let $\Gamma=\pi_{1}(X)$ denote the fundamental group of $X$ and $h: \Gamma \rightarrow \mathbf{Z}, h(\gamma)=$ $\operatorname{lk}(\gamma, K)$, the canonical projection. There is a short exact splitting sequence

$$
1 \longrightarrow \Gamma^{\prime} \longrightarrow \Gamma \longrightarrow\langle t \mid-\rangle \longrightarrow 1,
$$

where $\Gamma^{\prime}=[\Gamma, \Gamma]$ denotes the commutator subgroup of $\Gamma$. The surjection is given by $\gamma \mapsto t^{h(\gamma)}$. Hence $\Gamma$ is isomorphic to the semi-direct product $\Gamma^{\prime} \rtimes \mathbf{Z}$. Note that $\Gamma^{\prime}$ is the fundamental group of the infinite cyclic covering $X_{\infty}$ of $X$. The abelian group $\Gamma^{\prime} / \Gamma^{\prime \prime} \cong H_{1}\left(X_{\infty} ; \mathbf{Z}\right)$ becomes a $\mathbf{Z}\left[t^{ \pm 1}\right]$-module via the action of the group of covering transformations which is isomorphic to $\langle t \mid-\rangle$. The $\mathbf{Z}\left[t^{ \pm 1}\right]$-module $H_{1}\left(X_{\infty} ; \mathbf{Z}\right)$ is a finitely generated torsion module called the Alexander module of $K$. There are isomorphisms of $\mathbf{Z}\left[t^{ \pm 1}\right]$-modules

$$
H_{q}\left(\Gamma ; \mathbf{Z}\left[t^{ \pm 1}\right]\right) \cong H_{q}\left(X ; \mathbf{Z}\left[t^{ \pm 1}\right]\right) \cong H_{q}\left(X_{\infty} ; \mathbf{Z}\right), \quad q=0,1,
$$

where $\Gamma$ acts on $\mathbf{Z}\left[t^{ \pm 1}\right]$ via $\gamma p(t)=t^{h(\gamma)} p(t)$ for all $\gamma \in \Gamma$ and $p(t) \in$ $\mathbf{Z}\left[t^{ \pm 1}\right]$. (See $[\mathbf{9}$, Chapter 5] for more details.) In what follows we are mainly interested in the complex version $\mathbf{C} \otimes \Gamma^{\prime} / \Gamma^{\prime \prime} \cong H_{1}\left(\Gamma ; \mathbf{C}\left[t^{ \pm 1}\right]\right)$ of the Alexander module. As $\mathbf{C}\left[t^{ \pm 1}\right]$ is a principal ideal domain, the Alexander module $H_{1}\left(\Gamma ; \mathbf{C}\left[t^{ \pm 1}\right]\right)$ decomposes into a direct sum of cyclic modules of the form $\mathbf{C}\left[t^{ \pm 1}\right] /(t-\alpha)^{k}, \alpha \in \mathbf{C}^{*} \backslash\{1\}$ i.e. there exist $\alpha_{1}, \ldots, \alpha_{s} \in \mathbf{C}^{*}$ such that

$$
H_{1}\left(\Gamma ; \mathbf{C}\left[t^{ \pm 1}\right]\right) \cong \tau_{\alpha_{1}} \oplus \cdots \oplus \tau_{\alpha_{s}}, \text { where } \tau_{\alpha_{j}}=\bigoplus_{i_{j}=1}^{n_{\alpha_{j}}} \mathbf{C}\left[t^{ \pm 1}\right] /\left(t-\alpha_{j}\right)^{r_{i_{j}}}
$$


denotes the $\left(t-\alpha_{j}\right)$-torsion of $H_{1}\left(\Gamma ; \mathbf{C}\left[t^{ \pm 1}\right]\right)$. A generator of the order ideal of $H_{1}\left(X_{\infty} ; \mathbf{C}\right)$ is called the Alexander polynomial $\Delta_{K}(t) \in \mathbf{C}\left[t^{ \pm 1}\right]$ of $K$ i.e. $\Delta_{K}(t)$ is the product

$$
\Delta_{K}(t)=\prod_{j=1}^{s} \prod_{i_{j}=1}^{n_{\alpha_{j}}}\left(t-\alpha_{j}\right)^{r_{j_{i}}}
$$

Notice that the Alexander polynomial is symmetric and is well defined up to multiplication by a unit $c t^{k}$ of $\mathbf{C}\left[t^{ \pm 1}\right], c \in \mathbf{C}^{*}, k \in \mathbf{Z}$. Moreover, $\Delta_{K}(1) \neq 0$ (see $[8]$ ), and hence the $(t-1)$-torsion of the Alexander module is trivial. In fact, it is well known that, up to multiplication by a unit, we can assume that $\Delta_{K}$ is normalized in the following way: $\Delta_{K} \in \mathbf{Z}[t] \subset \mathbf{C}\left[t^{ \pm 1}\right]$ is a polynomial with integer coefficients such that $\Delta_{K}(0) \neq 0$ and $\Delta_{K}(1)=1$ (see $\left.[8,8 . \mathrm{D}]\right)$.

For completeness we will state the next lemma which shows that the cohomology groups $H^{*}\left(X ; \mathbf{C}\left[t^{ \pm 1}\right] /(t-\alpha)^{k}\right)$ are determined by the Alexander module $H_{1}\left(\Gamma ; \mathbf{C}\left[t^{ \pm 1}\right]\right)$. Recall that the action of $\Gamma$ on $\mathbf{C}\left[t^{ \pm 1}\right] /(t-\alpha)^{k}$ is induced by $\gamma p(t)=t^{h(\gamma)} p(t)$.

Lemma 2.4. Let $K \subset M^{3}$ be a knot with exterior $X=\overline{M^{3} \backslash V(K)}$ and $\Gamma$ its fundamental group. Let $\alpha \in \mathbf{C}^{*}$ and let $\tau_{\alpha}=\oplus_{i=1}^{n_{\alpha}} \mathbf{C}\left[t^{ \pm 1}\right] /(t-\alpha)^{r_{i}}$ denote the $(t-\alpha)$-torsion of the Alexander module $H_{1}\left(\Gamma ; \mathbf{C}\left[t^{ \pm 1}\right]\right)$. Then if $\alpha=1$ we have that $\tau_{1}$ is trivial and

$$
H^{q}\left(X ; \mathbf{C}\left[t^{ \pm 1}\right] /(t-1)^{k}\right) \cong \begin{cases}\mathbf{C} & \text { for } q=0,1 \\ 0 & \text { for } q \geq 2\end{cases}
$$

Moreover, for $\alpha \neq 1$ we have:

$$
H^{q}\left(X ; \mathbf{C}\left[t^{ \pm 1}\right] /(t-\alpha)^{k}\right) \cong \begin{cases}0 & \text { for } q \neq 1,2 \\ \oplus_{i=1}^{n_{\alpha}} \mathbf{C}\left[t^{ \pm 1}\right] /(t-\alpha)^{\min \left(k, r_{i}\right)} & \text { for } q=1,2 .\end{cases}
$$

In particular, $H^{1}\left(\Gamma ; \mathbf{C}\left[t^{ \pm 1}\right] /(t-\alpha)^{k}\right) \neq 0$ if and only $H_{1}\left(\Gamma ; \mathbf{C}\left[t^{ \pm 1}\right]\right)$ has non-trivial $(t-\alpha)$-torsion i.e. if $\Delta_{K}(\alpha)=0$.

Proof: During this proof we put $\Lambda=\mathbf{C}\left[t^{ \pm 1}\right]$. Let $A$ be a $\Lambda$-module, then by the extension of scalars $[\mathbf{6}$, III.3] we have an isomorphism

$$
H^{q}(X ; A) \cong H^{q}\left(\operatorname{Hom}_{\Lambda}\left(C_{*}\left(X_{\infty} ; \mathbf{C}\right) ; A\right)\right) .
$$

Since $\Lambda$ is a principal ideal domain, we can apply the universal coefficient theorem and obtain

$$
H^{q}(X ; A) \cong \operatorname{Ext}_{\Lambda}^{1}\left(H_{q-1}\left(X_{\infty} ; \mathbf{C}\right), A\right) \oplus \operatorname{Hom}_{\Lambda}\left(H_{q}\left(X_{\infty} ; \mathbf{C}\right), A\right) .
$$


Now $H_{0}\left(X_{\infty} ; \mathbf{C}\right) \cong \mathbf{C} \cong \Lambda /(t-1)$ and $H_{k}\left(X_{\infty} ; \mathbf{C}\right)=0$ for $k \geq 2$ (see $[\mathbf{8}$, Proposition 8.16]). Therefore,

$$
\begin{aligned}
& H^{0}(X ; A) \cong \operatorname{Hom}_{\Lambda}\left(H_{0}\left(X_{\infty} ; \mathbf{C}\right), A\right), \\
& H^{1}(X ; A) \cong \operatorname{Ext}_{\Lambda}^{1}\left(H_{0}\left(X_{\infty} ; \mathbf{C}\right), A\right) \oplus \operatorname{Hom}_{\Lambda}\left(H_{1}\left(X_{\infty} ; \mathbf{C}\right), A\right), \\
& H^{2}(X ; A) \cong \operatorname{Ext}_{\Lambda}^{1}\left(H_{1}\left(X_{\infty} ; \mathbf{C}\right), A\right) .
\end{aligned}
$$

To complete the proof, observe that for $\alpha, \beta \in \mathbf{C}^{*}$ and $k, l \in \mathbf{N}$ we have the following:

$$
\begin{gathered}
\operatorname{Hom}_{\Lambda}\left(\Lambda /(t-\alpha)^{k}, \Lambda /(t-\beta)^{l}\right) \cong \begin{cases}0 & \text { if } \alpha \neq \beta, \\
\Lambda /(t-\alpha)^{m} & \text { if } \alpha=\beta,\end{cases} \\
\operatorname{Ext}_{\Lambda}^{1}\left(\Lambda /(t-\alpha)^{k}, \Lambda /(t-\beta)^{l}\right) \cong \begin{cases}0 & \text { if } \alpha \neq \beta, \\
\Lambda /(t-\alpha)^{m} & \text { if } \alpha=\beta,\end{cases}
\end{gathered}
$$

where $m=\min \{k, l\}$ (see $[\mathbf{9}$, Proposition 2.4]). Notice that for $\beta \neq \alpha$, multiplication by $(t-\beta)$ induces an isomorphism of $\Lambda /(t-\alpha)^{k}$.

Corollary 2.5. Let $K \subset M^{3}$ be a knot and $\Gamma$ its group. Let $\alpha \in \mathbf{C}^{*}$ and let $\tau_{\alpha}=\oplus_{i=1}^{n_{\alpha}} \mathbf{C}\left[t^{ \pm 1}\right] /(t-\alpha)^{r_{i}}$ denote the $(t-\alpha)$-torsion of the Alexander module $H_{1}\left(\Gamma ; \mathbf{C}\left[t^{ \pm 1}\right]\right)$. Then we have that

$$
H^{q}\left(\Gamma ; \mathbf{C}\left[t^{ \pm 1}\right] /(t-1)^{k}\right) \cong \begin{cases}\mathbf{C} & \text { for } q=0,1 \\ 0 & \text { for } q=2\end{cases}
$$

and, for $\alpha \neq 1$ we have:

$$
H^{q}\left(\Gamma ; \mathbf{C}\left[t^{ \pm 1}\right] /(t-\alpha)^{k}\right) \cong \begin{cases}0 & \text { for } q=0 \\ \oplus_{i=1}^{n_{\alpha}} \mathbf{C}\left[t^{ \pm 1}\right] /(t-\alpha)^{\min \left(k, r_{i}\right)} & \text { for } q=1\end{cases}
$$

Proof: This is an immediate consequence of Lemmas 2.3 and 2.4.

2.3. Representation variety. Let $\Gamma$ be a finitely generated group. The set of all homomorphisms of $\Gamma$ into $\mathrm{SL}(n)$ has the structure of an affine algebraic set (see [17] for details). In what follows this affine algebraic set will be denoted by $R(\Gamma, \mathrm{SL}(n))$ or simply by $R_{n}(\Gamma)$. Let $\rho: \Gamma \rightarrow \mathrm{SL}(n)$ be a representation. The Lie algebra $\mathfrak{s l}(n)$ becomes a $\Gamma$-module via Ad $\circ$. This module will be simply denoted by $\mathfrak{s l}(n)_{\rho}$. A 1 -cocycle or derivation $d \in Z^{1}\left(\Gamma ; \mathfrak{s l}(n)_{\rho}\right)$ is a map $d: \Gamma \rightarrow \mathfrak{s l}(n)$ satisfying

$$
d\left(\gamma_{1} \gamma_{2}\right)=d\left(\gamma_{1}\right)+\operatorname{Ad} \circ \rho\left(\gamma_{1}\right)\left(d\left(\gamma_{2}\right)\right), \quad \forall \gamma_{1}, \gamma_{2} \in \Gamma .
$$

It was observed by André Weil [19] that there is a natural inclusion of the Zariski tangent space $T_{\rho}^{\mathrm{Zar}}\left(R_{n}(\Gamma)\right) \hookrightarrow Z^{1}\left(\Gamma ; \mathfrak{s l}(n)_{\rho}\right)$. Informally 
speaking, given a smooth curve $\rho_{\epsilon}$ of representations through $\rho_{0}=\rho$ one gets a derivation $d: \Gamma \rightarrow \mathfrak{s l}(n)$ by defining

$$
d(\gamma):=\left.\frac{d \rho_{\epsilon}(\gamma)}{d \epsilon}\right|_{\epsilon=0} \rho(\gamma)^{-1}, \quad \forall \gamma \in \Gamma .
$$

It is easy to see that the tangent space to the orbit by conjugation corresponds to the space of principal derivations $B^{1}\left(\Gamma ; \mathfrak{s l}(n)_{\rho}\right)$. Here, $b: \Gamma \rightarrow \mathfrak{s l}(n)$ is a principal derivation if there exists $x \in \mathfrak{s l}(n)$ such that $b(\gamma)=\operatorname{Ad} \circ \rho(\gamma)(x)-x$. A detailed account can be found in $[\mathbf{1 7}]$.

For the convenience of the reader, we state the following result which is implicitly contained in $[\mathbf{3}, \mathbf{1 4}, \mathbf{1 3}]$. A detailed proof of the following streamlined version can be found in $[\mathbf{1 2}]$ :

Proposition 2.6. Let $M$ be an orientable 3-manifold with infinite fundamental group $\pi_{1}(M)$ and incompressible torus boundary, and let $\rho: \pi_{1}(M) \rightarrow \operatorname{SL}(n)$ be a representation.

If $\operatorname{dim} H^{1}\left(\pi_{1}(M) ; \mathfrak{s l}(n)_{\rho}\right)=n-1$ then $\rho$ is a smooth point of the $\mathrm{SL}(n)$-representation variety $R_{n}\left(\pi_{1}(M)\right)$. More precisely, $\rho$ is contained in a unique component of dimension $n^{2}+n-2-\operatorname{dim} H^{0}\left(\pi_{1}(M) ; \mathfrak{s l}(n)_{\rho}\right)$.

\section{Reducible metabelian representations}

Recall that every nonzero complex number $\alpha \in \mathbf{C}^{*}$ determines an action of a knot group $\Gamma$ on the complex numbers given by $\gamma x=\alpha^{h(\gamma)} x$ for $\gamma \in \Gamma$ and $x \in \mathbf{C}$. The resulting $\Gamma$-module will be denoted by $\mathbf{C}_{\alpha}$. Notice that $\mathbf{C}_{\alpha}$ is isomorphic to $\mathbf{C}\left[t^{ \pm 1}\right] /(t-\alpha)$.

It is easy to see that a map $\Gamma \rightarrow \mathrm{GL}(2, \mathbf{C})$ given by

$$
\gamma \longmapsto\left(\begin{array}{cc}
1 & z_{1}(\gamma) \\
0 & 1
\end{array}\right)\left(\begin{array}{cc}
\alpha^{h(\gamma)} & 0 \\
0 & 1
\end{array}\right)=\left(\begin{array}{cc}
\alpha^{h(\gamma)} & z_{1}(\gamma) \\
0 & 1
\end{array}\right)
$$

is a representation if and only if the map $z_{1}: \Gamma \rightarrow \mathbf{C}_{\alpha}$ is a derivation i.e.

$$
\delta z_{1}\left(\gamma_{1}, \gamma_{2}\right)=\alpha^{h\left(\gamma_{1}\right)} z_{1}\left(\gamma_{2}\right)-z_{1}\left(\gamma_{1} \gamma_{2}\right)+z_{1}\left(\gamma_{1}\right)=0 \text { for all } \gamma_{1}, \gamma_{2} \in \Gamma \text {. }
$$

The representation given by (3) is non-abelian if and only if $\alpha \neq 1$ and the derivation $z_{1}$ is not a principal one. Hence it follows from Corollary 2.5 that such a reducible non-abelian representation exists if and only if $\alpha$ is a root of the Alexander polynomial. These representations were first studied independently by G. Burde [7] and G. de Rham [10].

We extend these considerations to a map $\Gamma \rightarrow \mathrm{GL}(3, \mathbf{C})$. It follows easily that

$$
\gamma \longmapsto\left(\begin{array}{ccc}
\alpha^{h(\gamma)} & z_{1}(\gamma) & z_{2}(\gamma) \\
0 & 1 & h(\gamma) \\
0 & 0 & 1
\end{array}\right)
$$


is a representation if and only if $\delta z_{1}=0$ and $\delta z_{2}+z_{1} \smile h=0$ i.e.

$$
\begin{cases}\delta z_{1}\left(\gamma_{1}, \gamma_{2}\right)=0 & \text { for all } \gamma_{1}, \gamma_{2} \in \Gamma, \\ \delta z_{2}\left(\gamma_{1}, \gamma_{2}\right)+z_{1}\left(\gamma_{1}\right) h\left(\gamma_{2}\right)=0 & \text { for all } \gamma_{1}, \gamma_{2} \in \Gamma .\end{cases}
$$

It was proved in $\left[\mathbf{4}\right.$, Theorem 3.2] that the 2-cocycle $z_{1} \smile h$ represents a non-trivial cohomology class in $H^{2}\left(\Gamma ; \mathbf{C}_{\alpha}\right)$ provided that $z_{1}$ is not a principal derivation and that the $(t-\alpha)$-torsion of the Alexander module is semi-simple i.e. $\tau_{\alpha}=\mathbf{C}\left[t^{ \pm 1}\right] /(t-\alpha) \oplus \cdots \oplus \mathbf{C}\left[t^{ \pm 1}\right] /(t-\alpha)$. Hence if we suppose that $z_{1}$ is not a principal derivation then it is clear that a non-abelian representation $\Gamma \rightarrow \mathrm{GL}(3, \mathbf{C})$ given by (4) can only exist if the $(t-\alpha)$-torsion $\tau_{\alpha}$ of the Alexander module has a direct summand of the form $\mathbf{C}\left[t^{ \pm 1}\right] /(t-\alpha)^{s}, s \geq 2$.

Representations $\Gamma \rightarrow \operatorname{GL}(n, \mathbf{C})$ of this type were studied in [16] where it was shown that the whole structure of the $(t-\alpha)$-torsion of the Alexander module can be recovered.

Let $\alpha \in \mathbf{C}^{*}$ be a non-zero complex number and $n \in \mathbf{Z}, n>1$. In what follows we consider the cyclic $\mathbf{C}\left[t^{ \pm 1}\right]$-module $\mathbf{C}\left[t^{ \pm 1}\right] /(t-\alpha)^{n-1}$ and the semi-direct product

$$
\mathbf{C}\left[t^{ \pm 1}\right] /(t-\alpha)^{n-1} \rtimes \mathbf{Z},
$$

where the multiplication is given by $\left(p_{1}, n_{1}\right)\left(p_{2}, n_{2}\right)=\left(p_{1}+t^{n_{1}} p_{2}, n_{1}+n_{2}\right)$. Let $I_{n} \in \mathrm{SL}(\mathrm{n})$ and $N_{n} \in \mathrm{GL}(n)$ denote the identity matrix and the upper triangular Jordan normal form of a nilpotent matrix of degree $n$ respectively. For later use we note the following lemma which follows easily from the Jordan normal form theorem:

Lemma 3.1. Let $\alpha \in \mathbf{C}^{*}$ be a nonzero complex number and let $\mathbf{C}^{n}$ be the $\mathbf{C}\left[t^{ \pm 1}\right]$-module with the action of $t^{k}$ given by

$$
t^{k} \mathbf{a}=\alpha^{k} \mathbf{a} J_{n}^{k},
$$

where $\mathbf{a} \in \mathbf{C}^{n}$ and $J_{n}=I_{n}+N_{n}$. Then the $\mathbf{C}\left[t^{ \pm 1}\right]$-module $\mathbf{C}^{n}$ is isomorphic to $\mathbf{C}\left[t^{ \pm 1}\right] /(t-\alpha)^{n}$.

There is a direct method to construct a reducible metabelian representation of $\mathbf{C}\left[t^{ \pm 1}\right] /(t-\alpha)^{n-1} \rtimes \mathbf{Z}$ into $\operatorname{GL}(n, \mathbf{C})$ (see [5, Proposition 3.13]). A direct calculation gives that

$$
(\mathbf{a}, 0) \longmapsto\left(\begin{array}{cc}
1 & \mathbf{a} \\
\mathbf{0} & I_{n-1}
\end{array}\right), \quad(0,1) \longmapsto\left(\begin{array}{cc}
\alpha & \mathbf{0} \\
\mathbf{0} & J_{n-1}^{-1}
\end{array}\right)
$$

defines a faithful representation $\mathbf{C}\left[t^{ \pm 1}\right] /(t-\alpha)^{n-1} \rtimes \mathbf{Z} \rightarrow \operatorname{GL}(n, \mathbf{C})$.

Note that the short exact splitting sequence (2) induces the sequence

$$
1 \longrightarrow \Gamma^{\prime} / \Gamma^{\prime \prime} \longrightarrow \Gamma / \Gamma^{\prime \prime} \longrightarrow\langle t \mid-\rangle \longrightarrow 1 \text {. }
$$


Hence $\Gamma / \Gamma^{\prime \prime}$ is isomorphic to the semi-direct product $\Gamma^{\prime} / \Gamma^{\prime \prime} \rtimes \mathbf{Z}$. Now, if $\rho: \Gamma \rightarrow \operatorname{GL}(n, \mathbf{C})$ is a metabelian representation, then $\rho$ factors through $\Gamma / \Gamma^{\prime \prime}$ and thus through the metabelian group $\Gamma^{\prime} / \Gamma^{\prime \prime} \rtimes \mathbf{Z}$.

Therefore, if the Alexander module $H_{1}\left(X_{\infty}, \mathbf{C}\right)$ has a direct summand of the form $\mathbf{C}\left[t^{ \pm 1}\right] /(t-\alpha)^{s}$ with $s \geq n-1 \geq 1$, we obtain a reducible, metabelian, non-abelian representation $\tilde{\varrho}: \Gamma \rightarrow \mathrm{GL}(n, \mathbf{C})$ as follows:

$$
\begin{aligned}
\tilde{\varrho}: \Gamma & \cong \Gamma^{\prime} \rtimes \mathbf{Z} \longrightarrow \Gamma^{\prime} / \Gamma^{\prime \prime} \rtimes \mathbf{Z} \longrightarrow\left(\mathbf{C} \otimes \Gamma^{\prime} / \Gamma^{\prime \prime}\right) \rtimes \mathbf{Z} \\
& \longrightarrow \mathbf{C}\left[t^{ \pm 1}\right] /(t-\alpha)^{s} \rtimes \mathbf{Z} \longrightarrow \mathbf{C}\left[t^{ \pm 1}\right] /(t-\alpha)^{n-1} \rtimes \mathbf{Z} \longrightarrow \operatorname{GL}(n, \mathbf{C})
\end{aligned}
$$

and given by

$$
\tilde{\varrho}(\gamma)=\left(\begin{array}{cc}
1 & \tilde{\mathbf{z}}(\gamma) \\
0 & I_{n-1}
\end{array}\right)\left(\begin{array}{cc}
\alpha^{h(\gamma)} & 0 \\
0 & J_{n-1}^{-h(\gamma)}
\end{array}\right)
$$

It is easy to see that a map $\tilde{\varrho}: \Gamma \rightarrow \operatorname{GL}(n)$ given by $(6)$ is a homomorphism if and only if $\tilde{\mathbf{z}}: \Gamma \rightarrow \mathbf{C}^{n-1}$ is a derivation i.e. for all $\gamma_{1}, \gamma_{2} \in \Gamma$ we have

$$
\tilde{\mathbf{z}}\left(\gamma_{1} \gamma_{2}\right)=\tilde{\mathbf{z}}\left(\gamma_{1}\right)+\alpha^{h\left(\gamma_{1}\right)} \tilde{\mathbf{z}}\left(\gamma_{2}\right) J_{n-1}^{h\left(\gamma_{1}\right)}
$$

For a better description of the cocycle $\tilde{\mathbf{z}}$, we introduce the following notations: for $m, k \in \mathbf{Z}, k \geq 0$, we define

$$
h_{k}(\gamma):=\left(\begin{array}{c}
h(\gamma) \\
k
\end{array}\right), \quad \text { where } \quad\left(\begin{array}{c}
m \\
k
\end{array}\right):= \begin{cases}\frac{m(m-1) \cdots(m-k+1)}{k !} & \text { if } k>0 \\
1 & \text { if } k=0\end{cases}
$$

Observe that if $m, k \in \mathbf{Z}$ and $0 \leq m<k$ then $\left(\begin{array}{c}m \\ k\end{array}\right)=0$.

It follows directly from the properties of the binomial coefficients that for each $k \in \mathbf{Z}, k \geq 0$, the cochains $h_{k} \in C^{1}(\Gamma ; \mathbf{C})$ are defined and satisfy:

$$
\delta h_{k}+\sum_{i=1}^{k-1} h_{i} \smile h_{k-i}=0 .
$$

Lemma 3.2. Let $\tilde{\mathbf{z}}: \Gamma \rightarrow \mathbf{C}^{n-1}$ be a map satisfying (7). The components $\tilde{z}_{k}: \Gamma \rightarrow \mathbf{C}_{\alpha}, 1 \leq k \leq n-1$, of $\tilde{\mathbf{z}}$ satisfy the equations

$$
\delta \tilde{z}_{k}+\sum_{i=1}^{k-1} h_{i} \smile \tilde{z}_{k-i}=0 .
$$

In particular $\tilde{z}_{1}: \Gamma \rightarrow \mathbf{C}_{\alpha}$ is a derivation. 
Proof: Note that $h_{0} \equiv 1, h_{1}=h, J_{n-1}^{m}=\left(I_{n-1}+N_{n-1}\right)^{m}=\sum_{i \geq 0}\left(\begin{array}{c}m \\ i\end{array}\right) N_{n-1}^{i}$, and $\left(x_{1}, \ldots, x_{n-1}\right) J_{n-1}^{m}=\left(x_{1}^{\prime}, x_{2}^{\prime}, \ldots, x_{n-1}^{\prime}\right)$ where

$$
x_{k}^{\prime}=\sum_{i=0}^{k-1}\left(\begin{array}{c}
m \\
i
\end{array}\right) x_{k-i}=x_{k}+\sum_{i=1}^{k-1}\left(\begin{array}{c}
m \\
i
\end{array}\right) x_{k-i} .
$$

It follows from this formula that $\tilde{\mathbf{z}}\left(\gamma_{1} \gamma_{2}\right)=\tilde{\mathbf{z}}\left(\gamma_{1}\right)+\alpha^{h\left(\gamma_{1}\right)} \tilde{\mathbf{z}}\left(\gamma_{2}\right) J_{n-1}^{h\left(\gamma_{1}\right)}$ holds if and only if for $k=1, \ldots, n-1$ we have

$$
\tilde{z}_{k}\left(\gamma_{1} \gamma_{2}\right)=\tilde{z}_{k}\left(\gamma_{1}\right)+\alpha^{h\left(\gamma_{1}\right)} \tilde{z}_{k}\left(\gamma_{2}\right)+\sum_{i=1}^{k-1} h_{i}\left(\gamma_{1}\right) \alpha^{h\left(\gamma_{1}\right)} \tilde{z}_{k-i}\left(\gamma_{2}\right) \text {. }
$$

In other words $0=\delta \tilde{z}_{k}+\sum_{i=1}^{k-1} h_{i} \smile \tilde{z}_{k-i}$ holds.

From now on we will suppose that, for $\alpha \in \mathbf{C}^{*} \backslash\{1\}$, the $(t-\alpha)$-torsion of the Alexander module is cyclic of the form

$$
\tau_{\alpha}=\mathbf{C}\left[t^{ \pm 1}\right] /(t-\alpha)^{n-1}, \quad \text { where } n \geq 2 .
$$

This is equivalent to requiring that $\alpha$ is a root of the Alexander polynomial $\Delta_{K}(t)$ of multiplicity $n-1$ and that $\operatorname{dim} H^{1}\left(\Gamma ; \mathbf{C}_{\alpha}\right)=1$ (see Corollary 2.5$)$.

Remark 3.3. Notice that by Blanchfield-duality [11, Chapter 7] the $(t-$ $\left.\alpha^{-1}\right)$-torsion of the Alexander module $H_{1}\left(\Gamma ; \mathbf{C}\left[t^{ \pm 1}\right]\right)$ is also of the form

$$
\tau_{\alpha^{-1}}=\mathbf{C}\left[t^{ \pm 1}\right] /\left(t-\alpha^{-1}\right)^{n-1} .
$$

More precisely, the Alexander polynomial $\Delta_{K}(t)$ is symmetric and hence $\alpha^{-1}$ is also a root of $\Delta_{K}(t)$ of multiplicity $n-1$ and $\operatorname{dim} H^{1}\left(\Gamma ; \mathbf{C}_{\alpha^{-1}}\right)=1$.

Let $\tilde{\varrho}: \Gamma \rightarrow \operatorname{GL}(n)$ be a representation given by (6) i.e. for all $\gamma \in \Gamma$ we have

$$
\tilde{\varrho}(\gamma)=\left(\begin{array}{cc}
1 & \tilde{\mathbf{z}}(\gamma) \\
0 & I_{n-1}
\end{array}\right)\left(\begin{array}{cc}
\alpha^{h(\gamma)} & 0 \\
0 & J_{n-1}^{-h(\gamma)}
\end{array}\right) .
$$

We will say that $\tilde{\varrho}$ can be upgraded to a representation into $\operatorname{GL}(n+1, \mathbf{C})$ if there is a cochain $\tilde{z}_{n}: \Gamma \rightarrow \mathbf{C}_{\alpha}$ such that the map $\Gamma \rightarrow \mathrm{GL}(n+1, \mathbf{C})$ given by

$$
\gamma \longmapsto\left(\begin{array}{cc}
1 & \left(\tilde{\mathbf{z}}(\gamma), \tilde{z}_{n}(\gamma)\right) \\
0 & I_{n}
\end{array}\right)\left(\begin{array}{cc}
\alpha^{h(\gamma)} & 0 \\
0 & J_{n}^{-h(\gamma)}
\end{array}\right)
$$

is a representation.

Lemma 3.4. Suppose that the $(t-\alpha)$-torsion of the Alexander module is cyclic of the form $\tau_{\alpha}=\mathbf{C}\left[t^{ \pm 1}\right] /(t-\alpha)^{n-1}, n \geq 2$ and let $\tilde{\varrho}: \Gamma \rightarrow$ $\mathrm{GL}(n, \mathbf{C})$ be a representation given by $(6)$.

Then $\varrho$ cannot be upgraded to a representation into $\mathrm{GL}(n+1, \mathbf{C})$ unless $\tilde{z}_{1}: \Gamma \rightarrow \mathbf{C}_{\alpha}$ is a principal derivation. 
Proof: By Lemma 3.1, the $\mathbf{C}\left[t^{ \pm 1}\right]$-module $\mathbf{C}^{n-1}$ with the action given by $t \mathbf{a}=\alpha \mathbf{a} J_{n-1}$ is isomorphic to $\mathbf{C}\left[t^{ \pm 1}\right] /(t-\alpha)^{n-1}$. Hence it follows from the universal coefficient theorem that, for $l \geq n-1$, we have:

$$
\begin{aligned}
H^{1}\left(\Gamma ; \mathbf{C}\left[t^{ \pm 1}\right] /(t-\alpha)^{l}\right) & \cong \operatorname{Hom}_{\mathbf{C}\left[t^{ \pm 1}\right]}\left(H_{1}\left(\Gamma ; \mathbf{C}\left[t^{ \pm 1}\right]\right), \mathbf{C}\left[t^{ \pm 1}\right] /(t-\alpha)^{l}\right) \\
& \cong \operatorname{Hom}_{\mathbf{C}\left[t^{ \pm 1}\right]}\left(\mathbf{C}\left[t^{ \pm 1}\right] /(t-\alpha)^{n-1}, \mathbf{C}\left[t^{ \pm 1}\right] /(t-\alpha)^{l}\right) \\
& \cong \mathbf{C}\left[t^{ \pm 1}\right] /(t-\alpha)^{n-1} .
\end{aligned}
$$

Hence if $l>n-1$ then every derivation $\tilde{z}: \Gamma \rightarrow \mathbf{C}\left[t^{ \pm 1}\right] /(t-\alpha)^{l}$, given by $\tilde{z}(\gamma)=\left(\tilde{z}_{1}(\gamma), \ldots, \tilde{z}_{l}(\gamma)\right)$ is cohomologous to a derivation for which the first $l-n+1$ components vanish. This proves the conclusion of the lemma.

Notice that the unipotent matrices $J_{n}$ and $J_{n}^{-1}$ are similar: a direct calculation shows that $P_{n} J_{n} P_{n}^{-1}=J_{n}^{-1}$ where $P_{n}=\left(p_{i j}\right), p_{i j}=$ $(-1)^{j}\left(\begin{array}{l}j \\ i\end{array}\right)$. The matrix $P_{n}$ is upper triangular with \pm 1 in the diagonal and $P_{n}^{2}$ is the identity matrix, and therefore $P_{n}=P_{n}^{-1}$.

Hence $\varrho$ is conjugate to a representation $\varrho: \Gamma \rightarrow \mathrm{GL}(n, \mathbf{C})$ given by (10)

$$
\varrho(\gamma)=\left(\begin{array}{cc}
\alpha^{h(\gamma)} & z(\gamma) \\
0 & J_{n-1}^{h(\gamma)}
\end{array}\right)=\left(\begin{array}{ccccc}
\alpha^{h(\gamma)} & z_{1}(\gamma) & z_{2}(\gamma) & \ldots & z_{n-1}(\gamma) \\
0 & 1 & h_{1}(\gamma) & \ldots & h_{n-2}(\gamma) \\
\vdots & \ddots & \ddots & \ddots & \vdots \\
\vdots & & \ddots & 1 & h_{1}(\gamma) \\
0 & \ldots & \ldots & 0 & 1
\end{array}\right),
$$

where $\mathbf{z}=\left(z_{1}, \ldots, z_{n-1}\right): \Gamma \rightarrow \mathbf{C}^{n-1}$ satisfies

$$
\mathbf{z}\left(\gamma_{1} \gamma_{2}\right)=\alpha^{h\left(\gamma_{1}\right)} \mathbf{z}\left(\gamma_{2}\right)+\mathbf{z}\left(\gamma_{1}\right) J_{n-1}^{h\left(\gamma_{2}\right)} .
$$

It follows directly that $\mathbf{z}(\gamma)=\tilde{\mathbf{z}}(\gamma) P_{n-1} J_{n-1}^{h(\gamma)}$ and in particular $z_{1}=-\tilde{z}_{1}$.

The same argument as in the proof of Lemma 3.2 shows that the cochains $z_{k}: \Gamma \rightarrow \mathbf{C}_{\alpha}$ satisfy:

$$
\delta z_{k}+\sum_{i=1}^{k-1} z_{i} \smile h_{k-i}=0 \quad \text { for } k=1, \ldots, n-1 .
$$

Therefore, the representation $\varrho: \Gamma \rightarrow \operatorname{GL}(n, \mathbf{C})$ can be upgraded into a representation $\Gamma \rightarrow \operatorname{GL}(n+1, \mathbf{C})$ if and only if $\sum_{i=1}^{n-1} z_{i} \smile h_{n-i}$ is a principal derivation.

Hence we obtain the following: 
Proposition 3.5. Suppose that the $(t-\alpha)$-torsion of the Alexander module is cyclic of the form $\tau_{\alpha}=\mathbf{C}\left[t^{ \pm 1}\right] /(t-\alpha)^{n-1}, n \geq 2$. Let $\tilde{\varrho}, \varrho: \Gamma \rightarrow$ $\mathrm{GL}(n, \mathbf{C})$ be the representations given by (6) and (10) respectively where $\tilde{z}_{1}=-z_{1}: \Gamma \rightarrow \mathbf{C}_{\alpha}$ is a non-principal derivation. Then the representations $\tilde{\varrho}$ and $\varrho$ cannot be upgraded to representations $\Gamma \rightarrow \mathrm{GL}(n+1, \mathbf{C})$ i.e. the cocycles

$$
\sum_{i=1}^{n-1} h_{i} \smile \tilde{z}_{n-i} \quad \text { and } \quad \sum_{i=1}^{n-1} z_{i} \smile h_{n-i}
$$

represent nontrivial cohomology classes in $H^{2}\left(\Gamma ; \mathbf{C}_{\alpha}\right)$.

Proof: The proposition follows from Lemma 3.4 and the above considerations.

Corollary 3.6. Suppose that the $(t-\alpha)$-torsion of the Alexander module is cyclic of the form $\tau_{\alpha}=\mathbf{C}\left[t^{ \pm 1}\right] /(t-\alpha)^{n-1}, n \geq 2$. Then

$$
\operatorname{dim} H^{2}\left(\Gamma ; \mathbf{C}_{\alpha \pm 1}\right)=1 \text {. }
$$

Proof: Proposition 3.5 implies that $\operatorname{dim} H^{2}\left(\Gamma ; \mathbf{C}_{\alpha^{ \pm 1}}\right) \geq 1$, and by Lemmas 2.4 and 2.3 we obtain the claimed result.

Example 3.7. Let us consider the knots $3_{1}, 8_{10}$, and $8_{20}$ in $S^{3}$. Their Alexander polynomials are given by $\Delta_{3_{1}}(t)=t^{2}-t+1, \Delta_{8_{20}}(t)=$ $\left(t^{2}-t+1\right)^{2}$, and $\Delta_{8_{10}}(t)=\left(t^{2}-t+1\right)^{3}$. In each case the Alexander module is cyclic.

A presentation of $\Gamma_{3_{1}}$ is given by $\Gamma_{3_{1}}=\langle S, T \mid S T S=T S T\rangle$. The knots $8_{10}$ and $8_{20}$ can be realized as the closures of $\hat{\sigma}=8_{10}$ and $\hat{\tau}=8_{20}$ of the braids $\sigma=\sigma_{1}^{-1} \sigma_{2}^{2} \sigma_{1}^{-2} \sigma_{2}^{3}$ and $\tau=\sigma_{1}^{3} \sigma_{2} \sigma_{1}^{-3} \sigma_{2}$ in the braid group $B_{3}$ on three strands. This gives the following presentations for the knot groups:

$$
\begin{aligned}
& \Gamma_{8_{10}}=\left\langle x_{1}, x_{2}, x_{3} \mid x_{1}=\sigma\left(x_{1}\right), x_{2}=\sigma\left(x_{2}\right)\right\rangle, \\
& \Gamma_{8_{20}}=\left\langle y_{1}, y_{2}, y_{3} \mid y_{1}=\tau\left(y_{1}\right), y_{2}=\tau\left(y_{2}\right)\right\rangle .
\end{aligned}
$$

All our computer supported calculations were carried out by using SageMath [18]. Moreover, we made a worksheet which contains the calculations and more details (available at http://mat.uab.cat/pubmat).

We let $\alpha$ denote the primitive 6 -th root of unity. For the trefoil knot a non-abelian reducible representation (10) is given by

$$
\varrho_{3_{1}}(S)=\left(\begin{array}{ll}
\alpha & 0 \\
0 & 1
\end{array}\right) \text { and } \varrho_{3_{1}}(T)=\left(\begin{array}{ll}
\alpha & 1 \\
0 & 1
\end{array}\right) \text {. }
$$


Notice that $\varrho_{3_{1}}$ can not be upgraded to a representation into $\operatorname{GL}(3, \mathbf{C})$ since $\alpha$ is a simple root of $\Delta_{3_{1}}$. This follows from Proposition 3.5 or from direct calculation (see also the worksheet).

For the knot $8_{20}$ a non-principal derivation $z_{1}: \Gamma_{8_{20}} \rightarrow \mathbf{C}_{\alpha}$ is given by $z_{1}\left(y_{1}\right)=0, z_{1}\left(y_{2}\right)=z_{1}\left(y_{3}\right)=1$. Thus we obtain the reducible metabelian representation $\varrho_{820}^{(2)}: \Gamma_{8_{20}} \rightarrow \mathrm{GL}(2, \mathbf{C})$ given by

$$
\varrho_{8_{20}}^{(2)}\left(y_{1}\right)=\left(\begin{array}{cc}
\alpha & 0 \\
0 & 1
\end{array}\right), \quad \varrho_{8_{20}}^{(2)}\left(y_{2}\right)=\left(\begin{array}{cc}
\alpha & 1 \\
0 & 1
\end{array}\right), \quad \varrho_{8_{20}}^{(2)}\left(y_{3}\right)=\left(\begin{array}{cc}
\alpha & 1 \\
0 & 1
\end{array}\right) .
$$

This representation can be upgraded to the representation $\varrho_{8_{20}}^{(3)}: \Gamma_{8_{20}} \rightarrow$ $\operatorname{GL}(3, \mathbf{C})$ given by $\varrho_{8_{20}}^{(3)}\left(y_{i}\right)=A_{i}$ where

$$
A_{1}=\left(\begin{array}{ccc}
\alpha & 0 & 0 \\
0 & 1 & 1 \\
0 & 0 & 1
\end{array}\right), \quad A_{2}=\left(\begin{array}{ccc}
\alpha & 1 & 0 \\
0 & 1 & 1 \\
0 & 0 & 1
\end{array}\right), \quad A_{3}=\left(\begin{array}{ccc}
\alpha & 1 & \alpha+1 \\
0 & 1 & 1 \\
0 & 0 & 1
\end{array}\right)
$$

Proposition 3.5 or computer supported calculations (see the worksheet) show that $\varrho_{8_{20}}^{(3)}$ can not be upgraded to a representation into $\mathrm{GL}(4, \mathbf{C})$.

Similarly, for the knot $8_{10}$ the representations $\varrho_{8_{10}}^{(2)}$ and $\varrho_{8_{10}}^{(3)}$ can be upgraded but $\varrho_{8_{10}}^{(4)}$ cannot (see the worksheet). The representation $\varrho_{8_{10}}^{(4)}$ : $\Gamma_{8_{10}} \rightarrow \mathrm{GL}(4, \mathbf{C})$ is given by $\varrho_{8_{10}}^{(4)}\left(x_{i}\right)=B_{i}$ where

$$
\begin{gathered}
B_{1}=\left(\begin{array}{cccc}
\alpha & 0 & 0 & 0 \\
0 & 1 & 1 & 0 \\
0 & 0 & 1 & 1 \\
0 & 0 & 0 & 1
\end{array}\right), \quad B_{2}=\left(\begin{array}{cccc}
\alpha & 1 & 0 & 0 \\
0 & 1 & 1 & 0 \\
0 & 0 & 1 & 1 \\
0 & 0 & 0 & 1
\end{array}\right), \\
B_{3}=\left(\begin{array}{cccc}
\alpha & 1 & \alpha-2 & \alpha+3 \\
0 & 1 & 1 & 0 \\
0 & 0 & 1 & 1 \\
0 & 0 & 0 & 1
\end{array}\right) .
\end{gathered}
$$

\section{Cohomological computations}

We suppose throughout this section that $K \subset M^{3}$ is a knot in a three dimensional integer homology sphere $M^{3}$ and that the $(t-\alpha)$-torsion of its Alexander module is cyclic of the form $\tau_{\alpha}=\mathbf{C}\left[t^{ \pm 1}\right] /(t-\alpha)^{n-1}$, $n \geq 2$, where $\alpha \in \mathbf{C}^{*}$ is a nonzero complex number. Let $\varrho: \Gamma \rightarrow \mathrm{GL}(n)$ be a representation given by (10) where $z_{1}: \Gamma \rightarrow \mathbf{C}_{\alpha}$ is a non-principal 
derivation:

$$
\varrho(\gamma)=\left(\begin{array}{cc}
\alpha^{h(\gamma)} & z(\gamma) \\
0 & J_{n-1}^{h(\gamma)}
\end{array}\right)=\left(\begin{array}{ccccc}
\alpha^{h(\gamma)} & z_{1}(\gamma) & z_{2}(\gamma) & \ldots & z_{n-1}(\gamma) \\
0 & 1 & h_{1}(\gamma) & \ldots & h_{n-2}(\gamma) \\
\vdots & \ddots & \ddots & \ddots & \vdots \\
\vdots & & \ddots & 1 & h_{1}(\gamma) \\
0 & \ldots & \ldots & 0 & 1
\end{array}\right)
$$

We choose an $n$-th root $\lambda$ of $\alpha$ and we define a reducible metabelian representation $\varrho_{\lambda}: \Gamma \rightarrow \mathrm{SL}(n)$ by

$$
\varrho_{\lambda}(\gamma)=\lambda^{-h(\gamma)} \varrho(\gamma) \text {. }
$$

The aim of the following sections is to calculate the first cohomology groups of $\Gamma$ with coefficients in the Lie algebra $\mathfrak{s l}(n) \operatorname{Ad} \circ \varrho_{\lambda}$. Notice that the action of $\Gamma$ via $A d \circ \varrho$ and $A d \circ \varrho_{\lambda}$ preserve $\mathfrak{s l}(n)$ and coincide since the center of $\operatorname{GL}(n)$ is the kernel of $\mathrm{Ad}: \operatorname{GL}(n) \rightarrow \operatorname{Aut}(\mathfrak{g l}(n))$. Hence we have the following isomorphisms of $\Gamma$-modules:

$$
\mathfrak{s l}(n)_{\mathrm{Ad} \circ \varrho_{\lambda}} \cong \mathfrak{s l}(n)_{\mathrm{Ad} \circ \varrho} \text { and } \mathfrak{g l}(n)_{\mathrm{Ad} \circ \varrho}=\mathfrak{s l}(n)_{\mathrm{Ad} \circ \varrho} \oplus \mathbf{C} I_{n},
$$

where $\Gamma$ acts trivially on the center $\mathbf{C} I_{n}$ of $\mathfrak{g l}(n)$. We will prove the following result:

Proposition 4.1. Let $K \subset M^{3}$ be a knot and suppose that the $(t-\alpha)$-torsion of the Alexander module of $K$ is of the form $\tau_{\alpha}=\mathbf{C}\left[t^{ \pm 1}\right] /(t-\alpha)^{n-1}$. Then for the representation $\varrho_{\lambda}: \Gamma \rightarrow \operatorname{SL}(n)$ we have $H^{0}\left(\Gamma ; \mathfrak{s l}(n){\operatorname{Ad} \circ \varrho_{\lambda}}\right)=$ 0 and

$$
\operatorname{dim} H^{1}\left(\Gamma ; \mathfrak{s l}(n)_{\operatorname{Ad} \circ \varrho_{\lambda}}\right)=n-1 .
$$

Notice that Propositions 4.1 and 2.6 will prove the first part of Theorem 1.1. The proof of Proposition 4.1 will occupy the rest of this section.

Example 4.2. Proposition 4.1 applies to the representations $\varrho_{3_{1}}^{(2)}, \varrho_{8_{20}}^{(3)}$, and $\varrho_{8_{10}}^{(4)}$. Therefore, the corresponding SL $(n)$-representations are smooth points of the representation variety, and are limits of irreducible representations.

Computer supported calculations for $8_{20}$ show that

$$
\operatorname{dim} H^{1}\left(\Gamma ; \mathfrak{s l}(2){ }_{\operatorname{Ad} \circ \varrho_{8_{20}}^{(2)}}\right)=2 .
$$

Similar calculations for $8_{10}$ give

$$
\operatorname{dim} H^{1}\left(\Gamma ; \mathfrak{s l}(2)_{\operatorname{Ad} \circ \varrho_{8_{10}}^{(2)}}\right)=2 \quad \text { and } \quad \operatorname{dim} H^{1}\left(\Gamma ; \mathfrak{s l}(3)_{\operatorname{Ad} \circ \varrho_{8_{10}}^{(3)}}\right)=3
$$

(see the worksheet). Therefore, Proposition 4.1 does not apply for the corresponding $\operatorname{SL}(n)$-representations. However, the representations $\varrho_{8_{20}}^{(2)}$ 
and $\varrho_{8_{10}}^{(2)}$ factor through surjections $\pi_{1}: \Gamma_{8_{10}} \rightarrow \Gamma_{3_{1}}$ and $\pi_{2}: \Gamma_{8_{20}} \rightarrow \Gamma_{3_{1}}$ respectively. These surjections are defined by $\pi_{1}\left(x_{1}\right)=T, \pi_{1}\left(x_{2}\right)=S$, $\pi_{1}\left(x_{3}\right)=T$, and $\pi_{2}\left(y_{1}\right)=S, \pi_{2}\left(y_{2}\right)=\pi_{2}\left(y_{3}\right)=T$ as indicated in Figure 1 (see also the worksheet). Hence, $\varrho_{8_{20}}^{(2)}$ and $\varrho_{8_{10}}^{(2)}$ are limits of irreducible representations. See also $[\mathbf{2}]$.
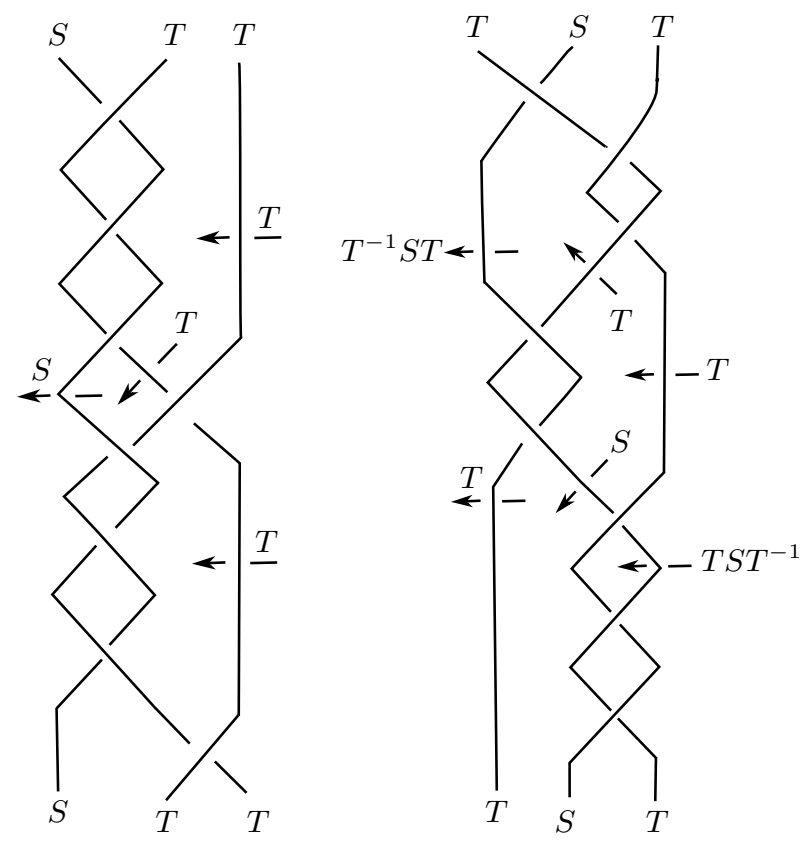

FiguRE 1. The surjections $\pi_{1}: \Gamma_{8_{10}} \rightarrow \Gamma_{3_{1}}$ and $\pi_{2}: \Gamma_{820} \rightarrow \Gamma_{3_{1}}$.

Throughout this section we will consider $\mathfrak{g l}(n)$ as a $\Gamma$-module via Ad $\varrho \varrho$ and for simplicity we will write $\mathfrak{g l}(n)$ for $\mathfrak{g l}(n)_{\mathrm{Ad} \circ \varrho}$. It follows from Equation (12) that

$$
H^{*}(\Gamma ; \mathfrak{g l}(n)) \cong H^{*}(\Gamma ; \mathfrak{s l}(n)) \oplus H^{*}(\Gamma ; \mathbf{C}) .
$$

In order to compute the first cohomology groups $H^{*}(\Gamma, \mathfrak{g l}(n))$ and describe the cocycles, we will construct and use an adequate filtration of the coefficient algebra $\mathfrak{g l}(n)$.

4.1. The setup. Let $\left(E_{1}, \ldots, E_{n}\right)$ denote the canonical basis of the space of column vectors. Hence $E_{i}^{j}:=E_{i} \cdot{ }^{t} E_{j}, 1 \leq i, j \leq n$, form the canonical basis of $\mathfrak{g l}(n)$. 
Note that for $A \in \mathrm{GL}(n), \operatorname{Ad}_{A}\left(E_{i}^{j}\right)=\left(A E_{i}\right)\left({ }^{t} E_{j} A^{-1}\right)$. The Lie algebra $\mathfrak{g l}(n)$ turns into a $\Gamma$-module via Ado@ i.e. for all $\gamma \in \Gamma$ we have

$$
\gamma \cdot E_{i}^{j}=\left(\varrho(\gamma) E_{i}\right)\left({ }^{t} E_{j} \varrho\left(\gamma^{-1}\right)\right) .
$$

Explicitly we have

$$
\begin{aligned}
\gamma \cdot E_{1}^{1} & =\left(\begin{array}{c}
\alpha^{h(\gamma)} \\
0 \\
\vdots \\
0
\end{array}\right)\left(\alpha^{-h(\gamma)}, z_{1}\left(\gamma^{-1}\right), \ldots, z_{n-1}\left(\gamma^{-1}\right)\right) \\
& =E_{1}^{1}+\alpha^{h(\gamma)} z_{1}\left(\gamma^{-1}\right) E_{1}^{2}+\cdots+\alpha^{h(\gamma)} z_{n-1}\left(\gamma^{-1}\right) E_{1}^{n}
\end{aligned}
$$

for $1<k \leq n$ :

$$
\gamma \cdot E_{1}^{k}=\alpha^{h(\gamma)} E_{1}^{k}+\alpha^{h(\gamma)} h_{1}\left(\gamma^{-1}\right) E_{1}^{k+1}+\cdots+\alpha^{h(\gamma)} h_{n-k}\left(\gamma^{-1}\right) E_{1}^{n} \text {; }
$$

$$
\gamma \cdot E_{k}^{1}=\left(\begin{array}{c}
z_{k-1}(\gamma) \\
h_{k-2}(\gamma) \\
\vdots \\
h_{1}(\gamma) \\
1 \\
0 \\
\vdots
\end{array}\right)\left(\alpha^{-h(\gamma)}, z_{1}\left(\gamma^{-1}\right), \ldots, z_{n-1}\left(\gamma^{-1}\right)\right)
$$

and for $1<i, j \leq n$ :

$$
\gamma \cdot E_{i}^{j}=\left(\begin{array}{c}
z_{i-1}(\gamma) \\
h_{i-2}(\gamma) \\
\vdots \\
h_{1}(\gamma) \\
1 \\
0 \\
\vdots
\end{array}\right)\left(0, \ldots, 0,1, h_{1}\left(\gamma^{-1}\right), \ldots, h_{n-j}\left(\gamma^{-1}\right)\right)
$$

For a given family $\left(X_{i}\right)_{i \in I}, X_{i} \in \mathfrak{g l}(n)$, we let $\left\langle X_{i} \mid i \in I\right\rangle \subset \mathfrak{g l}(n)$ denote the subspace of $\mathfrak{g l}(n)$ generated by the family. 
Remark 4.3. A first consequence of these calculations is that if $c \in$ $C^{1}(\Gamma ; \mathbf{C})$ is a cochain, then for $2 \leq i \leq n$ and $1 \leq j \leq n$ we have:

$\delta^{\mathfrak{g l}}\left(c E_{i}^{j}\right)=(\delta c) E_{i}^{j}+\left(h_{1} \smile c\right) E_{i-1}^{j}+\cdots+\left(h_{i-2} \smile c\right) E_{2}^{j}+\left(z_{i-1} \smile c\right) E_{1}^{j}+x$, where $x: \Gamma \times \Gamma \rightarrow\left\langle E_{k}^{l} \mid 1 \leq k \leq i, j<l \leq n\right\rangle$ is a 2-cochain. Here $\delta^{\mathfrak{g l}}$ and $\delta$ denote the coboundary operators of $C^{1}(\Gamma ; \mathfrak{g l}(n))$ and $C^{1}(\Gamma ; \mathbf{C})$ respectively.

In what follows we will also make use of the following $\Gamma$-modules: for $0 \leq i \leq n-1$, we define $C(i)=\left\langle E_{k}^{l} \mid 1 \leq k \leq n, n-i \leq l \leq n\right\rangle$. We have

$$
C(i)=\left\{\left(\begin{array}{cccccc}
0 & \ldots & 0 & c_{1, n-i} & \ldots & c_{1, n} \\
0 & \ldots & 0 & c_{2, n-i} & \ldots & c_{2, n} \\
\vdots & \vdots & \vdots & \vdots & & \vdots \\
0 & \ldots & 0 & c_{n-1, n-i} & \ldots & c_{n-1, n} \\
0 & \ldots & 0 & c_{n, n-i} & \ldots & c_{n, n}
\end{array}\right): c_{i, j} \in \mathbf{C}\right\}
$$

and $\mathfrak{g l}(n)=C(n-1) \supset C(n-2) \supset \cdots \supset C(0)=\left\langle E_{1}^{n}, \ldots, E_{n}^{n}\right\rangle \supset$ $C(-1)=0$.

We will denote by $X+C(i) \in C(k) / C(i)$ the class represented by $X \in C(k), 0 \leq i<k \leq n-1$.

4.2. Cohomology with coefficients in $\boldsymbol{C}(\boldsymbol{i})$. The aim of this subsection is to prove that for $0 \leq i \leq n-2$ the cohomology groups $H^{q}(\Gamma ; C(i)), 0 \leq q \leq 2$, vanish (see Proposition 4.8). First we will prove this for $i=0$ and in order to conclude we will apply the isomorphism $C(0) \cong C(i) / C(i-1)$ (see Lemma 4.6). Finally Lemma 4.7 permits us to compute a certain Bockstein operator.

Lemma 4.4. The vector space $\left\langle E_{1}^{n}\right\rangle$ is a submodule of $C(0)$ and thus of $\mathfrak{g l}(n)=C(n-1)$ and we have

$$
H^{0}\left(\Gamma ;\left\langle E_{1}^{n}\right\rangle\right)=0, \operatorname{dim} H^{1}\left(\Gamma ;\left\langle E_{1}^{n}\right\rangle\right)=\operatorname{dim} H^{2}\left(\Gamma ;\left\langle E_{1}^{n}\right\rangle\right)=1 .
$$

More precisely, the cocycles $z_{1} E_{1}^{n} \in Z^{1}\left(\Gamma ;\left\langle E_{1}^{n}\right\rangle\right)$ and

$$
\left(\sum_{i=1}^{n-1} z_{i} \smile h_{n-i}\right) E_{1}^{n} \in Z^{2}\left(\Gamma ;\left\langle E_{1}^{n}\right\rangle\right)
$$

represent generators of $H^{1}\left(\Gamma ;\left\langle E_{1}^{n}\right\rangle\right)$ and $H^{2}\left(\Gamma ;\left\langle E_{1}^{n}\right\rangle\right)$ respectively.

Proof: The isomorphism $\left\langle E_{1}^{n}\right\rangle \cong \mathbf{C}_{\alpha}$, Corollary 2.5, and Corollary 3.6 imply the dimension formulas. The form of the generating cocycles follows from the isomorphism $\left\langle E_{1}^{n}\right\rangle \cong \mathbf{C}_{\alpha}$ and Proposition 3.5. 
Lemma 4.5. The $\Gamma$-module $C(0) /\left\langle E_{1}^{n}\right\rangle$ is isomorphic to $\mathbf{C}\left[t^{ \pm 1}\right] /(t-$ $1)^{n-1}$. In particular, we obtain:

(1) for $q=0,1 \operatorname{dim} H^{q}\left(\Gamma ; C(0) /\left\langle E_{1}^{n}\right\rangle\right)=1$ and $H^{2}\left(\Gamma ; C(0) /\left\langle E_{1}^{n}\right\rangle\right)=0$,

(2) the vector $E_{2}^{n}$ represents a generator of $H^{0}\left(\Gamma ; C(0) /\left\langle E_{1}^{n}\right\rangle\right)$ and the cochain $\bar{v}_{1}: \Gamma \rightarrow C(0)$ given by

$$
\bar{v}_{1}(\gamma)=h_{1}(\gamma) E_{n}^{n}+h_{2}(\gamma) E_{n-1}^{n}+\cdots+h_{n-2}(\gamma) E_{2}^{n}
$$

represents a generator of $H^{1}\left(\Gamma ; C(0) /\left\langle E_{1}^{n}\right\rangle\right)$.

Proof: First notice that $C(0) /\left\langle E_{1}^{n}\right\rangle$ is a $(n-1)$-dimensional vector space. More precisely, a basis of this space is represented by the elements

$$
E_{n}^{n}, E_{n-1}^{n}, \ldots, E_{2}^{n} \text {. }
$$

It follows from (17) that the action of $\Gamma$ on $C(0) /\left\langle E_{1}^{n}\right\rangle$ factors through $h: \Gamma \rightarrow \mathbf{Z}$. More precisely, we have for all $\gamma \in \Gamma$ such that $h(\gamma)=1$ and for all $0 \leq l \leq n-1$

$$
\gamma \cdot E_{n-l}^{n}=E_{n-l}^{n}+E_{n-l-1}^{n} .
$$

Here we used the fact that if $h(\gamma)=1$ then $h_{i}(\gamma)=0$ for all $2 \leq i \leq n-1$.

On the other hand

$$
\left(1=(t-1)^{0},(t-1), \ldots,(t-1)^{n-2}\right)
$$

represents a basis of $\mathbf{C}\left[t^{ \pm 1}\right] /(t-1)^{n-1}$ and we have for all $\gamma \in \Gamma$ such that $h(\gamma)=1$ :

$$
\gamma \cdot(t-1)^{l}=(t-1)^{l}+(t-1)^{l+1}+p,
$$

where $p \in(t-1)^{n-1} \mathbf{C}\left[t^{ \pm 1}\right]$ and $0 \leq l \leq n-2$. Hence the bijection

$$
\varphi:\left\{(t-1)^{l} \mid 0 \leq l \leq n-2\right\} \longrightarrow\left\{E_{n-l}^{n} \mid 0 \leq l \leq n-2\right\}
$$

given by $\varphi$ : $(t-1)^{l} \mapsto E_{n-l}^{n}, 0 \leq l \leq n-2$, induces an isomorphism of $\Gamma$-modules

$$
\varphi: \mathbf{C}\left[t^{ \pm 1}\right] /(t-1)^{n-1} \stackrel{\cong}{\longrightarrow} C(0) /\left\langle E_{1}^{n}\right\rangle .
$$

Now, the first assertion follows from Corollary 2.5.

Moreover, it follows from the above considerations that $E_{2}^{n}$ represents a generator of $H^{0}\left(\Gamma ; C(0) /\left\langle E_{1}^{n}\right\rangle\right)$. To prove the second assertion consider the following short exact sequence

$$
0 \longrightarrow \mathbf{C}\left[t^{ \pm 1}\right] /(t-1)^{n-2} \stackrel{(t-1) \cdot}{\longrightarrow} \mathbf{C}\left[t^{ \pm 1}\right] /(t-1)^{n-1} \longrightarrow \mathbf{C} \longrightarrow 0
$$


which gives the following long exact sequence in cohomology:

$$
\begin{aligned}
0 \longrightarrow H^{0}\left(\Gamma ; \mathbf{C}\left[t^{ \pm 1}\right] /(t-1)^{n-2}\right) \stackrel{\cong}{\longrightarrow} H^{0}\left(\Gamma ; \mathbf{C}\left[t^{ \pm 1}\right] /(t-1)^{n-1}\right) \\
\longrightarrow H^{0}(\Gamma ; \mathbf{C}) \stackrel{\beta^{0}}{\longrightarrow} H^{1}\left(\Gamma ; \mathbf{C}\left[t^{ \pm 1}\right] /(t-1)^{n-2}\right) \\
\longrightarrow H^{1}\left(\Gamma ; \mathbf{C}\left[t^{ \pm 1}\right] /(t-1)^{n-1}\right) \stackrel{\cong}{\longrightarrow} H^{1}(\Gamma ; \mathbf{C}) \\
\longrightarrow H^{2}\left(\Gamma ; \mathbf{C}\left[t^{ \pm 1}\right] /(t-1)^{n-2}\right)=0 .
\end{aligned}
$$

The isomorphisms and the vanishing of $H^{2}\left(\Gamma ; \mathbf{C}\left[t^{ \pm 1}\right] /(t-1)^{n-2}\right)$ follow directly from Corollary 2.5.

Hence the Bockstein operator $\beta^{0}$ is an isomorphism: the element $e_{0}=$ $1 \in \mathbf{C}\left[t^{ \pm 1}\right] /(t-1)^{n-1}$ projects onto a generator of $H^{0}(\Gamma ; \mathbf{C})$ and if $\delta^{n-1}$ denotes the coboundary operator of $C^{*}\left(\Gamma ; \mathbf{C}\left[t^{ \pm 1}\right] /(t-1)^{n-1}\right)$ we obtain:

$$
\begin{aligned}
\delta^{n-1}\left(e_{0}\right)(\gamma) & =(\gamma-1) \cdot e_{0} \\
& =h_{1}(\gamma) e_{1}+h_{2}(\gamma) e_{2}+\cdots+h_{n-2}(\gamma) e_{n-1} \\
& =(t-1) \cdot\left(h_{1}(\gamma) e_{0}+h_{2}(\gamma) e_{1}+\cdots+h_{n-2}(\gamma) e_{n-2}\right)
\end{aligned}
$$

Hence the cocycle $\gamma \mapsto h_{1}(\gamma) e_{0}+h_{2}(\gamma) e_{1}+\cdots+h_{n-2}(\gamma) e_{n-2}$ represents a generator of $H^{1}\left(\Gamma ; \mathbf{C}\left[t^{ \pm 1}\right] /(t-1)^{n-2}\right)$. To conclude, recall that the isomorphism $\mathbf{C}\left[t^{ \pm 1}\right] /(t-1)^{n-1} \cong C(0) /\left\langle E_{1}^{n}\right\rangle$ is induced by the map $\varphi: e_{l} \mapsto E_{n-l}^{n}, 0 \leq l \leq n-2$.

Lemma 4.6. For $i \in \mathbf{Z}, 0 \leq i \leq n-3$, the $\Gamma$-module $C(i+1) / C(i)$ is isomorphic to $C(0)$.

Proof: It follows from (17) that, for all $i \in \mathbf{Z}, 0 \leq i \leq n-2$, the bijection

$$
\phi:\left\{E_{n-j}^{n-(i+1)}+C(i) \mid 0 \leq j \leq n-1\right\} \longrightarrow\left\{E_{n-j}^{n} \mid 0 \leq j \leq n-1\right\}
$$

given by $\phi\left(E_{n-j}^{n-(i+1)}+C(i)\right)=E_{n-j}^{n}$ induces an isomorphism of $\Gamma$-modules $\phi: C(i+1) / C(i) \rightarrow C(0)$.

Let us recall the definition of the cochains $h_{i} \in C^{1}(\Gamma ; \mathbf{C})$, given by $h_{i}(\gamma)=\left(\begin{array}{c}h(\gamma) \\ i\end{array}\right)$ (see Equation $\left.(8)\right)$. Recall also that for $1 \leq i \leq n-1$ the cochains $h_{i} \in C^{1}(\Gamma ; \mathbf{C})$ satisfy Equation $(9)$ :

$$
\delta h_{i}+\sum_{j=1}^{i-1} h_{j} \smile h_{i-j}=0 .
$$


Lemma 4.7. Let $\delta^{\mathfrak{g l}}$ denote the coboundary operator of $C^{*}(\Gamma ; \mathfrak{g l}(n))$. Then for all $0 \leq k \leq n-2$ there exists a cochain $x_{k-1} \in C^{2}(\Gamma ; C(k-1))$ such that

$$
\delta^{\mathfrak{g l}}\left(\sum_{i=2}^{n} h_{n-i+1} E_{i}^{n-k}\right)=\left(\sum_{i=1}^{n-1} z_{i} \smile h_{n-i}\right) E_{1}^{n-k}+x_{k-1} .
$$

Proof: Equation (17) and Remark 4.3 imply that

$$
\begin{aligned}
\delta^{\mathfrak{g l}}\left(h_{n-i+1} E_{i}^{n-k}\right)= & z_{i-1} \smile h_{n-i+1} E_{1}^{n-k} \\
& +\sum_{l=2}^{i-1} h_{i-l} \smile h_{n-i+1} E_{l}^{n-k}+\delta h_{n-i+1} E_{i}^{n-k}+x_{i, k-1},
\end{aligned}
$$

where $x_{i, k-1} \in C^{2}(\Gamma ; C(k-1))$ and $\delta$ is the boundary operator of $C^{*}(\Gamma ; \mathbf{C})$. Therefore,

$$
\begin{aligned}
\delta^{\mathfrak{g l}}\left(\sum_{i=2}^{n} h_{n-i+1} E_{i}^{n-k}\right)= & \left(\sum_{i=2}^{n} z_{i-1} \smile h_{n-i+1}\right) E_{1}^{n-k} \\
& +\sum_{i=2}^{n} \sum_{l=2}^{i-1} h_{i-l} \smile h_{n-i+1} E_{l}^{n-k} \\
& +\sum_{i=2}^{n} \delta h_{n-i+1} E_{i}^{n-k}+x_{k-1},
\end{aligned}
$$

where $x_{k-1}=\sum_{i=2}^{n} x_{i, k-1} \in C^{2}(\Gamma ; C(k-1))$, A direct calculation gives that

$$
\begin{aligned}
\sum_{i=2}^{n} \sum_{l=2}^{i-1} h_{i-l} \smile h_{n-i+1} E_{l}^{n-k} & =\sum_{l=2}^{n-1} \sum_{i=l+1}^{n} h_{i-l} \smile h_{n-i+1} E_{l}^{n-k} \\
& =\sum_{l=2}^{n-1}\left(\sum_{i=1}^{n-l} h_{i} \smile h_{n-l+1-i}\right) E_{l}^{n-k} .
\end{aligned}
$$

Thus

$$
\begin{aligned}
\delta^{\mathfrak{g l}}\left(h_{n-i+1} E_{i}^{n-k}\right)= & \left(\sum_{i=1}^{n-1} z_{i} \smile h_{n-i}\right) E_{1}^{n-k}+\delta h_{1} E_{n}^{n-k} \\
& +\sum_{i=1}^{n-2}\left(\delta h_{n-i}+\sum_{l=1}^{n-i-1} h_{l} \smile h_{n-i-l}\right) E_{i}^{n-k}+x_{k-1} .
\end{aligned}
$$

Now $\delta h_{1}=0$ and by (9) we have $\delta h_{n-i}+\sum_{l=1}^{n-i} h_{l} \smile h_{n-i+1-l}=0$. Hence we obtain the claimed formula. 
Proposition 4.8. For all $i \in \mathbf{Z}, 0 \leq i \leq n-2$, and $0 \leq q \leq 2$ we have

$$
H^{q}(\Gamma ; C(i))=0 .
$$

Proof: We start by proving the result for $i=0$. Consider the short exact sequence

$$
0 \longrightarrow\left\langle E_{1}^{n}\right\rangle \succ C(0) \longrightarrow C(0) /\left\langle E_{1}^{n}\right\rangle \longrightarrow 0 .
$$

As the $\mathbf{C}\left[t^{ \pm 1}\right]$-modules $\left\langle E_{1}^{n}\right\rangle$ and $\mathbf{C}_{\alpha} \cong \mathbf{C}\left[t^{ \pm 1}\right] /(t-\alpha)$ are isomorphic, the sequence (19) gives us a long exact sequence in cohomology:

$$
\begin{aligned}
0=H^{0}\left(\Gamma ;\left\langle E_{1}^{n}\right\rangle\right) \longrightarrow H^{0}(\Gamma ; C(0)) \longrightarrow H^{0}\left(\Gamma ; C(0) /\left\langle E_{1}^{n}\right\rangle\right) \\
\quad \stackrel{\beta_{0}^{0}}{\longrightarrow} H^{1}\left(\Gamma ;\left\langle E_{1}^{n}\right\rangle\right) \longrightarrow H^{1}(\Gamma ; C(0)) \longrightarrow H^{1}\left(\Gamma ; C(0) /\left\langle E_{1}^{n}\right\rangle\right) \\
\quad \stackrel{\beta_{0}^{1}}{\longrightarrow} H^{2}\left(\Gamma ;\left\langle E_{1}^{n}\right\rangle\right) \longrightarrow H^{2}(\Gamma ; C(0)) \longrightarrow H^{2}\left(\Gamma ; C(0) /\left\langle E_{1}^{n}\right\rangle\right)=0 .
\end{aligned}
$$

Here, for $q=0,1$, we denoted by $\beta_{0}^{q}: H^{q}\left(\Gamma ; C(0) /\left\langle E_{1}^{n}\right\rangle\right) \rightarrow H^{q+1}\left(\Gamma ;\left\langle E_{1}^{n}\right\rangle\right)$ the Bockstein homomorphism. By Lemma $4.5, E_{2}^{n}$ represents a generator of $H^{0}\left(\Gamma ; C(0) /\left\langle E_{1}^{n}\right\rangle\right)$, so

$$
\begin{aligned}
\beta_{0}^{0}\left(E_{2}^{n}\right)(\gamma) & =(\gamma-1) \cdot\left(E_{2}^{n}\right) \\
& =\gamma \cdot E_{2}^{n}-E_{2}^{n}=z_{1}(\gamma) E_{1}^{n}
\end{aligned}
$$

By Lemma $4.4, z_{1} E_{1}^{n}$ is a generator of $H^{1}\left(\Gamma ;\left\langle E_{1}^{n}\right\rangle\right)$, and by Lemma 4.5 $\operatorname{dim} H^{0}\left(\Gamma ; C(0) /\left\langle E_{1}^{n}\right\rangle\right)=1=\operatorname{dim} H^{1}\left(\Gamma ;\left\langle E_{1}^{n}\right\rangle\right)$, thus $\beta_{0}^{0}$ is an isomorphism. Consequently $H^{0}(\Gamma ; C(0))=0$ as $H^{0}\left(\Gamma ;\left\langle E_{1}^{n}\right\rangle\right)=0$ by Lemma 4.4 .

Now by Lemma 4.5 , the cochain $\bar{v}_{1}: \Gamma \rightarrow C(0)$ given by

$$
\bar{v}_{1}(\gamma)=h_{1}(\gamma) E_{n}^{n}+h_{2}(\gamma) E_{n-1}^{n}+\cdots+h_{n-1}(\gamma) E_{2}^{n}
$$

represents a generator of $H^{1}\left(\Gamma ; C(0) /\left\langle E_{1}^{n}\right\rangle\right)$ and by Lemma 4.7

$$
\beta_{0}^{1}\left(h_{1} E_{n}^{n}+h_{2} E_{n-1}^{n}+\cdots+h_{n-1} E_{2}^{n}\right)=\left(\sum_{i=1}^{n-1} z_{i} \smile h_{n-i}\right) E_{1}^{n} .
$$

Moreover, by Proposition 3.5 the cocycle $\left(\sum_{i=1}^{n-1} z_{i} \smile h_{n-i}\right) E_{1}^{n}$ represents a generator of $H^{2}\left(\Gamma ;\left\langle E_{1}^{n}\right\rangle\right)$. Thus $\beta_{0}^{1}$ is an isomorphism and $H^{q}(\Gamma ; C(0))=$ 0 for $q=1,2$.

Now suppose that $H^{q}\left(\Gamma ; C\left(i_{0}\right)\right)=0$ for $0 \leq i_{0} \leq n-3, q=0,1,2$, and consider the following short exact sequence of $\Gamma$-modules:

$$
0 \longrightarrow C\left(i_{0}\right) \longleftrightarrow C\left(i_{0}+1\right) \longrightarrow C\left(i_{0}+1\right) / C\left(i_{0}\right) \longrightarrow 0 .
$$


This sequence induces a long exact sequence in cohomology

$$
\begin{aligned}
& 0 \longrightarrow H^{0}\left(\Gamma ; C\left(i_{0}\right)\right) \longrightarrow H^{0}\left(\Gamma ; C\left(i_{0}+1\right)\right) \\
& \longrightarrow H^{0}\left(\Gamma ; C\left(i_{0}+1\right) / C\left(i_{0}\right)\right) \longrightarrow H^{1}\left(\Gamma ; C\left(i_{0}\right)\right) \longrightarrow H^{1}\left(\Gamma ; C\left(i_{0}+1\right)\right) \\
& \longrightarrow H^{1}\left(\Gamma ; C\left(i_{0}+1\right) / C\left(i_{0}\right)\right) \longrightarrow H^{2}\left(\Gamma ; C\left(i_{0}\right)\right) \longrightarrow H^{2}\left(\Gamma ; C\left(i_{0}+1\right)\right) \\
& \longrightarrow H^{2}\left(\Gamma ; C\left(i_{0}+1\right) / C\left(i_{0}\right)\right) \longrightarrow \cdots
\end{aligned}
$$

By Lemma 4.6 we have $C\left(i_{0}+1\right) / C\left(i_{0}\right) \cong C(0)$. Hence $H^{q}\left(\Gamma ; C\left(i_{0}+\right.\right.$ 1) $\left./ C\left(i_{0}\right)\right)=0$, and the hypothesis implies that $H^{q}\left(\Gamma ; C\left(i_{0}+1\right)\right) \cong$ $H^{q}\left(\Gamma ; C\left(i_{0}\right)\right)=0$ for $q=0,1,2$.

4.3. Cohomology with coefficients in $\mathfrak{g l}(\boldsymbol{n})$. In this subsection we will prove Proposition 4.1.

Proof of Proposition 4.1: In order to compute the dimensions of the cohomology groups $H^{q}(\Gamma ; \mathfrak{g l}(n)), q=0,1$, we consider the short exact sequence

$$
0 \longrightarrow C(n-2) \succ C(n-1)=\mathfrak{g l}(n) \longrightarrow \mathfrak{g l}(n) / C(n-2) \longrightarrow 0 .
$$

The sequence (21) gives rise to the following long exact cohomology sequence:

$$
\begin{aligned}
& 0 \longrightarrow H^{0}(\Gamma ; C(n-2)) \longrightarrow H^{0}(\Gamma ; \mathfrak{g l}(n)) \\
& \longrightarrow H^{0}(\Gamma ; \mathfrak{g l}(n) / C(n-2)) \longrightarrow H^{1}(\Gamma ; C(n-2)) \longrightarrow H^{1}(\Gamma ; \mathfrak{g l}(n)) \\
& \quad \longrightarrow H^{1}(\Gamma ; \mathfrak{g l}(n) / C(n-2)) \longrightarrow H^{2}(\Gamma ; C(n-2)) \longrightarrow \cdots
\end{aligned}
$$

As $H^{q}(\Gamma ; C(n-2))=0, q=0,1,2$, we conclude that

$$
H^{q}(\Gamma ; \mathfrak{g l}(n)) \cong H^{q}(\Gamma ; \mathfrak{g l}(n) / C(n-2)) \quad \text { for } q=0,1 .
$$

It remains to understand the quotient $\mathfrak{g l}(n) / C(n-2)$.

Clearly the vectors $E_{n}^{1}, \ldots, E_{1}^{1}$ represent a basis of $\mathfrak{g l}(n) / C(n-2)$ and there exists a $\Gamma$-module $A$ such that the following sequence

$$
0 \longrightarrow\left\langle E_{1}^{1}+C(n-2)\right\rangle \succ \mathfrak{g l}(n) / C(n-2) \longrightarrow A \longrightarrow 0
$$


is exact. Now the sequence (22) induces the following exact cohomology sequence:

$$
\begin{gathered}
0 \longrightarrow H^{0}\left(\Gamma ;\left\langle E_{1}^{1}+C(n-2)\right\rangle\right) \longrightarrow H^{0}(\Gamma ; \mathfrak{g l}(n) / C(n-2)) \longrightarrow H^{0}(\Gamma ; A) \\
\longrightarrow H^{1}\left(\Gamma ;\left\langle E_{1}^{1}+C(n-2)\right\rangle\right) \longrightarrow H^{1}(\Gamma ; \mathfrak{g l}(n) / C(n-2)) \\
\longrightarrow H^{1}(\Gamma ; A) \longrightarrow H^{2}\left(\Gamma ;\left\langle E_{1}^{1}+C(n-2)\right\rangle\right) \\
\longrightarrow H^{2}(\Gamma ; \mathfrak{g l}(n) / C(n-2)) \longrightarrow H^{2}(\Gamma ; A) \longrightarrow \cdots
\end{gathered}
$$

Observe that the action of $\Gamma$ on $\left\langle E_{1}^{1}+C(n-2)\right\rangle$ is trivial. Therefore, $\left\langle E_{1}^{1}+C(n-2)\right\rangle$ and $\mathbf{C}$ are isomorphic $\Gamma$-modules. By Corollary 2.5 we obtain

$$
\operatorname{dim} H^{q}\left(\Gamma ;\left\langle E_{1}^{1}+C(n-2)\right\rangle\right)=1 \quad \text { for } q=0,1
$$

and $H^{2}\left(\Gamma ;\left\langle E_{1}^{1}+C(n-2)\right\rangle\right)=0$.

To complete the proof we will make use of Lemma 4.9, which states that the $\Gamma$-module $A$ is isomorphic to $\mathbf{C}\left[t^{ \pm 1}\right] /\left(t-\alpha^{-1}\right)^{n-1}$. Recall that Lemma 2.4 implies that $H^{0}\left(\Gamma ; \mathbf{C}\left[t^{ \pm 1}\right] /\left(t-\alpha^{-1}\right)^{n-1}\right)=0$ and

$$
\operatorname{dim} H^{1}\left(\Gamma ; \mathbf{C}\left[t^{ \pm 1}\right] /\left(t-\alpha^{-1}\right)^{n-1}\right)=n-1 .
$$

Therefore, sequence (23) gives:

$$
H^{0}(\Gamma ; \mathfrak{g l}(n)) \cong H^{0}(\Gamma ; \mathfrak{g l}(n) / C(n-2)) \cong H^{0}(\Gamma ; \mathbf{C}) \cong \mathbf{C} .
$$

The short exact sequence

$$
\begin{aligned}
0 \longrightarrow H^{1}(\Gamma ; \mathbf{C}) & \longrightarrow H^{1}(\Gamma ; \mathfrak{g l}(n) / C(n-2)) \\
& \cong H^{1}(\Gamma ; \mathfrak{g l}(n)) \longrightarrow H^{1}(\Gamma ; A) \longrightarrow 0
\end{aligned}
$$

implies that $\operatorname{dim} H^{1}(\Gamma ; \mathfrak{g l}(n))=n$. The proposition follows now from Equation (13).

Lemma 4.9. The $\Gamma$-module $A$ is isomorphic to $\mathbf{C}\left[t^{ \pm 1}\right] /\left(t-\alpha^{-1}\right)^{n-1}$. Consequently

$$
H^{0}(\Gamma ; A)=0, \quad \operatorname{dim} H^{1}(\Gamma ; A)=n-1 .
$$

Proof of Lemma 4.9: The proof is similar to the proof of Lemma 4.5. As a $\mathbf{C}$-vector space the dimension of $A$ is $n-1$ and a basis is given by $\left(\overline{E_{n}^{1}}, \ldots, \overline{E_{2}^{1}}\right)$ where $\bar{E}_{i}^{1}=E_{i}^{1}+C(n-2) \in A$ is the class represented by $E_{i}^{1}, 2 \leq i \leq n$. In order to prove that $A$ is isomorphic to $\mathbf{C}\left[t^{ \pm 1}\right] /(t-$ $\left.\alpha^{-1}\right)^{n-1}$ observe that by (16)

$$
\gamma \cdot E_{k}^{1}=\alpha^{-h(\gamma)}\left(E_{k}^{1}+h_{1}(\gamma) E_{k-1}^{1}+\cdots+h_{k-2}(\gamma) E_{2}^{1}\right)+X_{k},
$$


where $X_{k} \in E_{1}^{1}+C(n-2)$. Therefore, the action of $\Gamma$ on $A$ factors through $h: \Gamma \rightarrow \mathbf{Z}$. More precisely, we have for all $\gamma \in \Gamma$ such that $h(\gamma)=1$

$$
\gamma \cdot \bar{E}_{k}^{1}=\alpha^{-1}\left(\bar{E}_{k}^{1}+\bar{E}_{k-1}^{1}\right) .
$$

On the other hand $e_{l}=\left(\alpha\left(t-\alpha^{-1}\right)\right)^{l}, 0 \leq l \leq n-2$, represents a basis of $\mathbf{C}\left[t^{ \pm 1}\right] /\left(t-\alpha^{-1}\right)^{n-1}$ and we have for all $\gamma \in \Gamma$ such that $h(\gamma)=1$ :

$$
\gamma \cdot e_{l}=\alpha^{-1}\left(e_{l}+e_{l+1}\right)+p \text {, where } p \in\left(t-\alpha^{-1}\right)^{n-1} \mathbf{C}\left[t^{ \pm 1}\right] .
$$

Hence the bijection $\psi:\left\{e_{l} \mid 0 \leq l \leq n-2\right\} \rightarrow\left\{\bar{E}_{k}^{1} \mid 2 \leq k \leq n\right\}$ given by $\varphi: e_{l} \mapsto \bar{E}_{n-l}^{1}, 0 \leq l \leq n-2$, induces an isomorphism of $\Gamma$-modules $\psi: \mathbf{C}\left[t^{ \pm 1}\right] /\left(t-\alpha^{-1}\right)^{n-1} \stackrel{\cong}{\cong} A$.

Finally, the dimension equations follow from Lemma 2.4 and Remark 3.3.

We obtain immediately that under the hypotheses of Proposition 4.1 the representation $\varrho_{\lambda}$ is a smooth point of the representation variety $R_{n}(\Gamma)$. This proves the first part of Theorem 1.1.

Proposition 4.10. Let $K \subset M^{3}$ be a knot in the homology sphere $M^{3}$. If the $(t-\alpha)$-torsion $\tau_{\alpha}$ of the Alexander module is cyclic of the form $\mathbf{C}\left[t, t^{-1}\right] /(t-\alpha)^{n-1}, n \geq 2$, then the representation $\varrho_{\lambda}$ is a smooth point of the representation variety $R_{n}(\Gamma)$; it is contained in a unique $\left(n^{2}+n-2\right)$-dimensional component $R_{\varrho_{\lambda}}$ of $R_{n}(\Gamma)$.

Proof: By Proposition 2.6 and Proposition 4.1, the representation $\varrho_{\lambda}$ is contained in a unique component $R_{\varrho_{\lambda}}$ of dimension $\left(n^{2}+n-2\right)$. Moreover,

$$
\begin{aligned}
\operatorname{dim} Z^{1}(\Gamma ; \mathfrak{s l}(n)) & =\operatorname{dim} H^{1}(\Gamma ; \mathfrak{s l}(n))+\operatorname{dim} B^{1}(\Gamma ; \mathfrak{s l}(n)) \\
& =(n-1)+\left(n^{2}-1\right) \\
& =n^{2}+n-2 .
\end{aligned}
$$

Hence the representation $\varrho_{\lambda}$ is a smooth point of $R_{n}(\Gamma)$ which is contained in an unique $\left(n^{2}+n-2\right)$-dimensional component $R_{\varrho_{\lambda}}$.

For later use, we describe more precisely the derivations $v_{k}: \Gamma \rightarrow$ $\mathfrak{s l}(n), 1 \leq k \leq n-1$, which represent a basis of $H^{1}(\Gamma ; \mathfrak{s l}(n))$.

Corollary 4.11. There exists cochains $z_{1}^{-}, \ldots, z_{n-1}^{-} \in C^{1}\left(\Gamma ; \mathbf{C}_{\alpha^{-1}}\right)$ such that $\delta z_{k}^{-}+\sum_{i=1}^{k-1} h_{i} \smile z_{k-i}^{-}=0$ for $k=1, \ldots, n-1$ and $z_{1}^{-}: \Gamma \rightarrow$ $\mathbf{C}_{\alpha^{-1}}$ is a non-principal derivation. 
Moreover, there exist cochains $g_{k}: \Gamma \rightarrow \mathbf{C}$ and $x_{k}: \Gamma \rightarrow \mathbf{C}(n-2)$, $1 \leq k \leq n-1$, such that the cochains $v_{k}: \Gamma \rightarrow \mathfrak{s l}(n)$ given by

$$
v_{k}=g_{k} E_{1}^{1}+z_{k}^{-} E_{2}^{1}+\cdots+z_{1}^{-} E_{k+1}^{1}+x_{k}
$$

are cocycles and represent a basis of $H^{1}(\Gamma ; \mathfrak{s l}(n))$.

Proof: Recall that the vector space $A$ admits as a basis the family $\left(\bar{E}_{n}^{1}, \ldots, \bar{E}_{2}^{1}\right)$ and that it is isomorphic to $\mathbf{C}\left[t^{ \pm 1}\right] /\left(t-\alpha^{-1}\right)^{n-1}$. Moreover it is easily seen that $A$ is isomorphic to the $\Gamma$-module of column vectors $\mathbf{C}^{n-1}$ where the action is given by $t^{k} a=\alpha^{-k} J_{n-1}^{k} a$. Hence a cochain $\mathbf{z}^{-}: \Gamma \rightarrow A$ with coordinates $\mathbf{z}^{-}={ }^{t}\left(z_{n-1}^{-}, \ldots, z_{1}^{-}\right)$is a cocycle in $Z^{1}(\Gamma ; A)$ if and only if for all $\gamma_{1}, \gamma_{2} \in \Gamma$

$$
\mathbf{z}^{-}\left(\gamma_{1} \gamma_{2}\right)=\mathbf{z}^{-}\left(\gamma_{1}\right)+\alpha^{-h\left(\gamma_{1}\right)} J_{n-1}^{h\left(\gamma_{1}\right)} \mathbf{z}^{-}\left(\gamma_{2}\right) .
$$

It follows, as in the proof of Lemma 3.2, that this is equivalent to

$$
z_{k}^{-}\left(\gamma_{1} \gamma_{2}\right)=z_{k}^{-}\left(\gamma_{1}\right)+\alpha^{-h\left(\gamma_{1}\right)} z_{k}^{-}\left(\gamma_{2}\right)+\sum_{i=1}^{k-1} h_{i}\left(\gamma_{1}\right) \alpha^{-h\left(\gamma_{1}\right)} z_{k-i}^{-}\left(\gamma_{2}\right) \text {. }
$$

In other words, for $1 \leq k \leq n-1$,

$$
0=\delta z_{k}^{-}+\sum_{i=1}^{k-1} h_{i} \smile z_{k-i}^{-} .
$$

By Remark 3.3, if $z_{1}^{-} \in Z^{1}\left(\Gamma ; \mathbf{C}_{\alpha^{-1}}\right)$ is a non-principal derivation, there exist cochains $z_{k}^{-}: \Gamma \rightarrow \mathbf{C}_{\alpha^{-1}}, 2 \leq k \leq n-1$, such that

$$
0=\delta z_{k}^{-}+\sum_{i=1}^{k-1} h_{i} \smile z_{k-i}^{-} .
$$

Consequently, as $\operatorname{dim} H^{1}(\Gamma ; A)=n-1$, the cochains

$$
\mathbf{z}_{k}^{-}=z_{k}^{-} \bar{E}_{2}^{1}+\cdots+z_{1}^{-} \bar{E}_{k+1}^{1}, \quad 1 \leq k \leq n-1,
$$

represent a basis of $H^{1}(\Gamma ; A)$. The proof is completed by noticing that the projection $H^{1}(\Gamma ; \mathfrak{g l}(n)) \rightarrow H^{1}(\Gamma ; A)$ restricts to an isomorphism between $H^{1}(\Gamma ; \mathfrak{s l}(n))$ and $H^{1}(\Gamma ; A)$.

\section{Irreducible $\mathrm{SL}(\boldsymbol{n})$ representations}

This section will be devoted to the proof of the last part of Theorem 1.1. In Proposition 4.10 we proved that the representation $\varrho_{\lambda}$ is a smooth point of $R_{n}(\Gamma)$ which is contained in a unique $\left(n^{2}+n-2\right)$-dimensional component $R_{\varrho_{\lambda}}$. Then, to prove the existence of irreducible representations in that component, we will make use of Corollary 4.11 and Burnside's theorem on matrix algebras. 
We start with the following technical lemma which is implicitly contained in $[\mathbf{1 4}, \S 2]$.

Lemma 5.1. Let $\Gamma$ be the knot group of $K \subset M^{3}$, and let $\varphi: \Gamma \rightarrow \mathbf{Z}$ be an epimorphism. Then there exists a presentation

$$
\Gamma \cong\left\langle S_{1}, \ldots, S_{k} \mid V_{1}, \ldots, V_{k-1}\right\rangle,
$$

such that $\varphi\left(S_{i}\right)=1$ for all $1 \leq i \leq k$.

Proof: Every presentation of $\Gamma$, obtained from a cell decomposition of $X=\overline{M^{3} \backslash V(K)}$, has deficiency one $[\mathbf{1 5}$, Chapter $\mathrm{V}]$, i.e. we have a presentation $\Gamma \cong\left\langle T_{1}, \ldots, T_{l} \mid W_{1}, \ldots, W_{l-1}\right\rangle$. We put $a_{i}=\varphi\left(T_{i}\right)$. Then the $\operatorname{gcd}\left\{a_{i} \mid 1 \leq i \leq k\right\}=1$ since $\varphi$ is surjective. Therefore we obtain $b_{i} \in \mathbf{Z}$ such that $1=\sum_{i=1}^{l} a_{i} b_{i}$, and $S=T_{1}^{b_{1}} T_{2}^{b_{2}} \cdots T_{l}^{b_{l}}$ maps under $\varphi$ to 1 . We define $S_{i}=T_{i} S^{1-a_{i}}$. We obtain a presentation

$\Gamma \cong\left\langle S, S_{1}, \ldots, S_{l}, T_{1}, \ldots, T_{l} \mid S^{-1} T_{1}^{b_{1}} \ldots T_{l}^{b_{l}}, S_{i} S^{a_{i}-1} T_{i}^{-1}, W_{1}, \ldots, W_{l-1}\right\rangle$,

and by Tietze transformations $\Gamma \cong\left\langle S, S_{1}, \ldots, S_{l} \mid V_{1}, \ldots, V_{l}\right\rangle$. Now, the deficiency of the latter presentation is one, and each generator maps to 1 under $\varphi$.

Proof of the last part of Theorem 1.1: To prove that the component $R_{\varrho_{\lambda}}$ contains irreducible non-metabelian representations, we will generalize the argument given in $[\mathbf{3}]$ for $n=3$.

By Lemma 5.1, we obtain a presentation of the knot group $\Gamma=$ $\left\langle S_{1}, \ldots, S_{k} \mid V_{1}, \ldots, V_{k-1}\right\rangle$ such that $h\left(S_{i}\right)=1$. This condition assures that each principal derivation $d: \Gamma \rightarrow \mathbf{C}_{\alpha}$ satisfies $d\left(S_{i}\right)=d\left(S_{j}\right)$ for all $1 \leq i, j \leq k$. Modulo conjugation of the representation $\varrho_{\lambda}$, we can assume that $z_{1}\left(S_{1}\right)=\cdots=z_{n-1}\left(S_{1}\right)=0$. This conjugation corresponds to adding a principal derivation to the cochains $z_{i}$, $1 \leq i \leq n-1$. We will also assume that the second generator $S_{2}$ verifies $z_{1}\left(S_{2}\right)=b_{1} \neq 0=z_{1}\left(S_{1}\right)$. This is always possible since $z_{1}$ is non-principal derivation. Hence

$$
\varrho_{\lambda}\left(S_{1}\right)=\alpha^{-1 / n}\left(\begin{array}{c|c}
\alpha & 0 \\
\hline 0 & J_{n-1}
\end{array}\right) \quad \text { and } \varrho_{\lambda}\left(S_{2}\right)=\alpha^{-1 / n}\left(\begin{array}{c|c}
\alpha & b \\
\hline 0 & J_{n-1}
\end{array}\right),
$$

where $b=\left(b_{1}, \ldots, b_{n-1}\right)$ with $b_{1} \in \mathbf{C}^{*}$ and $b_{i}=z_{i}\left(S_{2}\right) \in \mathbf{C}$ for $2 \leq i \leq$ $n-1$.

Let $v_{n-1} \in Z^{1}(\Gamma ; \mathfrak{s l}(n))$ be a cocycle such that:

$$
v_{n-1}=z_{1}^{-} E_{n}^{1}+z_{2}^{-} E_{n-1}^{1}+\cdots+z_{n-1}^{-} E_{2}^{1}+g_{n-1} E_{1}^{1}+x_{n-1}
$$


given by Corollary 4.11. Up to adding a principal derivation to the cocycle $z_{1}^{-}$we assume that $z_{1}^{-}\left(S_{1}\right)=0$. Notice that, the proof of Lemma 5.5 of $[3]$ generalizes to our situation, and hence $z_{1}^{-}\left(S_{2}\right) \neq 0$.

Let $\rho_{t}$ be a deformation of $\varrho_{\lambda}$ with leading term $v_{n-1}$ :

$$
\rho_{t}=\left(I_{n}+t v_{n-1}+o(t)\right) \varrho_{\lambda}, \text { where } \lim _{t \rightarrow 0} \frac{o(t)}{t}=0 .
$$

We may apply the following lemma (whose proof is completely analogous to that of Lemma 5.3 in $[\mathbf{3}])$ to this deformation for $A(t)=\rho_{t}\left(S_{1}\right)$.

Lemma 5.2. Let $\rho_{t}: \Gamma \rightarrow \operatorname{SL}(n)$ be a curve in $R_{n}(\Gamma)$ with $\rho_{0}=\varrho_{\lambda}$. Then there exists a curve $C_{t}$ in $\mathrm{SL}(n)$ such that $C_{0}=I_{n}$ and

$$
\operatorname{Ad}_{C_{t}} \circ \rho_{t}\left(S_{1}\right)=\left(\begin{array}{cccc}
a_{11}(t) & 0 & \ldots & 0 \\
0 & a_{22}(t) & \ldots & a_{2 n}(t) \\
\vdots & \vdots & & \vdots \\
0 & a_{n 2}(t) & \ldots & a_{n n}(t)
\end{array}\right)
$$

for all sufficiently small t.

Therefore, we may suppose that $a_{n 1}(t)=0$, and since

$$
a_{n 1}(t)=t \lambda^{n-1}\left(z_{1}^{-}\left(S_{1}\right)+\delta c\left(S_{1}\right)\right)+o(t), \text { for } c \in \mathbf{C},
$$

it follows that

$$
a_{n 1}^{\prime}(0)=\lambda^{n-1}\left(z_{1}^{-}\left(S_{1}\right)+\left(\alpha^{-1}-1\right) c\right)=0
$$

and hence $c=0$. For $B(t)=\rho_{t}\left(S_{2}\right)$, we obtain $b_{n 1}^{\prime}(0)=\lambda^{n-1} z_{1}^{-}\left(S_{2}\right) \neq 0$. Hence, we can apply the following technical lemma (whose proof will be postponed to the end of this section).

Lemma 5.3. Let $A(t)=\left(a_{i j}(t)\right)_{1 \leq i, j \leq n}$ and $B(t)=\left(b_{i j}(t)\right)_{1 \leq i, j \leq n}$ be matrices depending analytically on $t$ such that

$A(t)=\left(\begin{array}{c|c}a_{11}(t) & 0 \\ \hline 0 & A_{11}(t)\end{array}\right), \quad A(0)=\varrho_{\lambda}\left(S_{1}\right)=\alpha^{-1 / n}\left(\begin{array}{c|c}\alpha & 0 \\ \hline 0 & J_{n-1}\end{array}\right)$,

and

$$
B(0)=\varrho_{\lambda}\left(S_{2}\right)=\alpha^{-1 / n}\left(\begin{array}{c|c}
\alpha & b \\
\hline 0 & J_{n-1}
\end{array}\right) .
$$

If the first derivative $b_{n 1}^{\prime}(0) \neq 0$ then for sufficiently small $t, t \neq 0$, the matrices $A(t)$ and $B(t)$ generate the full matrix algebra $M(n, \mathbf{C})$.

Hence for sufficiently small $t \neq 0$ we obtain that $A(t)=\rho_{t}\left(S_{1}\right)$ and $B(t)=\rho_{t}\left(S_{2}\right)$ generate $M(n, \mathbf{C})$. By Burnside's matrix theorem, such a representation $\rho_{t}$ is irreducible. 
To conclude the proof of Theorem 1.1, we will prove that all irreducible representations sufficiently close to $\varrho_{\lambda}$ are non-metabelian. In order to do so, we will make use of the following result of $\mathrm{H}$. U. Boden and S. Friedl [5, Theorem 1.2]: for every irreducible metabelian representation $\rho: \Gamma \rightarrow \mathrm{SL}(n)$ we have $\operatorname{tr} \rho\left(S_{1}\right)=0$. Now, we have $\operatorname{tr} \varrho_{\lambda}\left(S_{1}\right)=\lambda^{-1}\left(\lambda^{n}+n-1\right)$ and we claim that $\lambda^{n}+n-1 \neq 0$. Notice that $\alpha=\lambda^{n}$ is a root of the normalized Alexander polynomial $\Delta_{K}$ and $\lambda^{n}+n-1=0$ would imply that $1-n$ is a root of $\Delta_{K}$. This in turn would imply that $t+n-1$ divides $\Delta_{K}(t)$ and hence $n$ divides $\Delta_{K}(1)=1$ which is impossible since $n \geq 2$. Therefore, $\operatorname{tr}\left(\rho\left(S_{1}\right)\right) \neq 0$ for all irreducible representations sufficiently close to $\varrho_{\lambda}$. This proves Theorem 1.1.

Remark 5.4. Let $\rho_{\lambda}: \Gamma \rightarrow \mathrm{SL}(n)$ be the diagonal representation given by $\rho_{\lambda}(\mu)=\operatorname{diag}\left(\lambda^{n-1}, \lambda^{-1} I_{n-1}\right)$ where $\mu$ is a meridian of $K$. The orbit $\mathcal{O}\left(\rho_{\lambda}\right)$ of $\rho_{\lambda}$ under the action of conjugation of $\mathrm{SL}(n)$ is contained in the closure $\overline{\mathcal{O}\left(\varrho_{\lambda}\right)}$. Hence $\varrho_{\lambda}$ and $\rho_{\lambda}$ project to the same point $\chi_{\lambda}$ of the variety of characters $X_{n}(\Gamma)=R_{n}(\Gamma) / / \mathrm{SL}(n)$.

It would be natural to study the local picture of the variety of characters $X_{n}(\Gamma)=R_{n}(\Gamma) / / \mathrm{SL}(n)$ at $\chi_{\lambda}$ as done in $[\mathbf{1 3}, \S 8]$. Unfortunately, there are much more technical difficulties since in this case the quadratic cone $Q\left(\rho_{\lambda}\right)$ coincides with the Zariski tangent space $Z^{1}\left(\Gamma ; \mathfrak{s l}(n)_{\rho_{\lambda}}\right)$. Therefore the third obstruction has to be considered.

Proof of Lemma 5.3: The proof follows exactly the proof of Proposition 5.4 in $[\mathbf{3}]$. We denote by $\mathcal{A}_{t} \subset \mathfrak{g l}(n)$ the algebra generated by $A(t)$ and $B(t)$. For any matrix $A$ we let $P_{A}(X)$ denote its characteristic polynomial. We have $P_{A_{11}(0)}=\left(\lambda^{-1}-X\right)^{n-1}$ and $a_{11}(0)=\lambda^{n-1}$. Since $\alpha=\lambda^{n} \neq 1$ we obtain $P_{A_{11}(0)}\left(a_{11}(0)\right) \neq 0$. It follows that $P_{A_{11}(t)}\left(a_{11}(t)\right) \neq 0$ for small $t$ and hence

$$
\begin{aligned}
\frac{1}{P_{A_{11}(t)}\left(a_{11}(t)\right)} P_{A_{11}(t)}(A(t)) & =\left(\begin{array}{c|c}
1 & 0 \\
\hline 0 & 0
\end{array}\right) \\
& =\left(\begin{array}{c}
1 \\
0 \\
\vdots \\
0
\end{array}\right) \otimes(1,0, \ldots, 0) \in \mathbf{C}[A(t)] \subset \mathcal{A}_{t} .
\end{aligned}
$$

In the next step we will prove that

$$
\mathcal{A}_{t}\left(\begin{array}{c}
1 \\
0 \\
\vdots \\
0
\end{array}\right)=\mathbf{C}^{n} \text { and }(1,0, \ldots, 0) \mathcal{A}_{t}=\mathbf{C}^{n} \text {, for small } t \in \mathbf{C}
$$


It follows from this that $\mathcal{A}_{t}$ contains all rank one matrices since a rank one matrix can be written as $v \otimes w$ where $v$ is a column vector and $w$ is a row vector. Note also that $A(v \otimes w)=(A v) \otimes w$ and $(v \otimes w) A=v \otimes(w A)$. Since each matrix is the sum of rank one matrices the proposition follows.

Now consider the vectors

$$
(1,0, \ldots, 0) A(0),(1,0, \ldots, 0) B(0), \ldots,(1,0, \ldots, 0) B(0)^{n-1} .
$$

Then for $1 \leq k \leq n-1$ :

$$
(1,0, \ldots, 0) B(0)^{k}=\lambda^{-k}\left(\alpha^{k}, b \sum_{j=0}^{k-1} \alpha^{k-1-j} J^{j}\right)
$$

and the dimension $D$ of the vector space

$$
\left\langle(1,0, \ldots, 0) A(0),(1,0, \ldots, 0) B(0), \ldots(1,0, \ldots, 0) B(0)^{n-1}\right\rangle
$$

is equal to

$$
\begin{aligned}
D & =\operatorname{dim}\left\langle(\alpha, 0),(\alpha, b),\left(\alpha^{2}, \alpha b+b J\right), \ldots,\left(\alpha^{n-1}, b \sum_{j=0}^{k-1} \alpha^{k-1-j} J^{j}\right)\right\rangle \\
& =\operatorname{dim}\left\langle(\alpha, 0),(0, b),(0, b J), \ldots,\left(0, b J^{n-2}\right)\right\rangle .
\end{aligned}
$$

Here, $J=J_{n-1}=I_{n-1}+N_{n-1}$ where $N_{n-1} \in G L(n-1, \mathbf{C})$ is the upper triangular Jordan normal form of a nilpotent matrix of degree $n-1$. Then a direct calculation gives that

$$
\operatorname{dim}\left\langle b, b J, \ldots, b J^{n-2}\right\rangle=\operatorname{dim}\left\langle b, b N, \ldots, b N^{n-2}\right\rangle=n-1, \text { as } b_{1} \neq 0 .
$$

Thus $\operatorname{dim}\left\langle(1,0, \ldots, 0) A(0),(1,0, \ldots, 0) B(0), \ldots(1,0, \ldots, 0) B(0)^{n-1}\right\rangle=$ $n$ and the vectors

$$
(1,0, \ldots, 0) A(0),(1,0, \ldots, 0) B(0), \ldots,(1,0, \ldots, 0) B(0)^{n-1}
$$

form a basis of the space of row vectors. This proves that $(1,0, \ldots, 0) \mathcal{A}_{t}$ is the space of row vectors for sufficiently small $t$.

In the final step consider the $n$ column vectors

$$
a_{1}(t)=A(t)\left(\begin{array}{c}
1 \\
0 \\
\vdots \\
0
\end{array}\right), a_{i+2}(t)=A^{i}(t) B(t)\left(\begin{array}{c}
1 \\
0 \\
\vdots \\
0
\end{array}\right), 0 \leq i \leq n-2
$$


and write $B(t)\left(\begin{array}{c}1 \\ 0 \\ \vdots \\ 0\end{array}\right)=\left(\begin{array}{c}b_{11}(t) \\ \mathbf{b}(t)\end{array}\right)$ for the first column of $B(t)$; then

$$
a_{1}(t)=\left(\begin{array}{c}
a_{11}(t) \\
\mathbf{0}
\end{array}\right), a_{i+2}(t)=A^{i}(t)\left(\begin{array}{c}
b_{11}(t) \\
\mathbf{b}(t)
\end{array}\right), 0 \leq i \leq n-2 .
$$

Define the function $f(t):=\operatorname{det}\left(a_{1}(t), \ldots, a_{n}(t)\right)$ and $g(t)$ by:

$$
f(t)=a_{11}(t) g(t) \text {, where } g(t)=\operatorname{det}\left(\mathbf{b}(t), A_{11}(t) \mathbf{b}(t), \ldots, A_{11}^{n-2}(t) \mathbf{b}(t)\right) .
$$

Now, for $k \geq 0$ the $k$-th derivative $g^{(k)}(t)$ of $g(t)$ is given by:

$$
\sum_{s_{1}, \ldots, s_{n-1} \geq 0} c_{s_{1}, \ldots, s_{n-1}} \operatorname{det}\left(\mathbf{b}^{\left(s_{1}\right)}(t),\left(A_{11}(t) \mathbf{b}(t)\right)^{\left(s_{2}\right)}, \ldots,\left(A_{11}^{n-2}(t) \mathbf{b}(t)\right)^{\left(s_{n-1}\right)}\right),
$$

where

$$
c_{s_{1}, \ldots, s_{n-1}}= \begin{cases}\left(\begin{array}{c}
k \\
s_{1}, \ldots, s_{n-1}
\end{array}\right)=\frac{k !}{s_{1} ! \cdots s_{n-1} !} & \text { if } s_{1}+\cdots+s_{n-1}=k \\
0 & \text { othewise. }\end{cases}
$$

As $\mathbf{b}(0)=0$ we obtain, for $0 \leq k \leq n-2, g^{(k)}(0)=0$ and consequently $f^{(k)}(0)=0$ for all $0 \leq k \leq n-2$.

Now, for $k=n-1$, we have

$$
\begin{aligned}
\frac{g^{(n-1)}(0)}{(n-1) !} & =\operatorname{det}\left(\mathbf{b}^{\prime}(0),\left(A_{11}(t) \mathbf{b}(t)\right)^{\prime}(0), \ldots,\left(A_{11}^{n-2}(t) \mathbf{b}(t)\right)^{\prime}(0)\right) \\
& =\operatorname{det}\left(\mathbf{b}^{\prime}(0), A_{11}(0) \mathbf{b}^{\prime}(0), \ldots, A_{11}^{n-2}(0) \mathbf{b}^{\prime}(0)\right) \\
& =\operatorname{det}\left(\mathbf{b}^{\prime}(0),\left(\lambda^{-1} J\right) \mathbf{b}^{\prime}(0), \ldots,\left(\lambda^{-1} J\right)^{n-2} \mathbf{b}^{\prime}(0)\right) \\
& =\operatorname{det}\left(\mathbf{b}^{\prime}(0), \lambda^{-1} N \mathbf{b}^{\prime}(0), \ldots, \lambda^{-(n-2)} N^{n-2} \mathbf{b}^{\prime}(0)\right) \\
& \neq 0 \text { since } b_{n 1}^{\prime} \neq 0 .
\end{aligned}
$$

Thus, $f^{(n-1)}(0)=a_{11}(0) g^{(n-1)}(0) \neq 0$ and $f(t) \neq 0$ for sufficiently small $t, t \neq 0$.

\section{References}

[1] L. Ben Abdelghani, Espace des représentations du groupe d'un noed classique dans un groupe de Lie, Ann. Inst. Fourier (Grenoble) 50(4) (2000), 1297-1321.

[2] L. Ben AbDelghani, Tangent cones and local geometry of the representation and character varieties of knot groups, Algebr. Geom. Topol. 10(1) (2010), 433-463. DOI: 10.2140/agt.2010.10.433. 
[3] L. Ben Abdelghani, M. Heusener, and H. Jebali, Deformations of metabelian representations of knot groups into $\mathrm{SL}(3, \mathbf{C}), J$. Knot Theory Ramifications 19(3) (2010), 385-404. DOI: 10.1142/ S0218216510007887.

[4] L. Ben AbDelghani And D. Lines, Involutions on knot groups and varieties of representations in a Lie group, J. Knot Theory Ramifications 11(1) (2002), 81-104. DOI: 10.1142/S0218216502001482.

[5] H. U. Boden And S. Friedl, Metabelian $\mathrm{SL}(n, \mathbb{C})$ representations of knot groups, Pacific J. Math. 238(1) (2008), 7-25. DOI: 10.2140/pjm.2008.238.7.

[6] K. S. Brown, "Cohomology of Groups", Graduate Texts in Mathematics 87, Springer-Verlag, New York-Berlin, 1982.

[7] G. Burde, Darstellungen von Knotengruppen, Math. Ann. 173(1) (1967), 24-33. DOI : $10.1007 /$ BF01351516.

[8] G. Burde, Z. Heiner, And M. Heusener, "Knots", 3rd fully revised and extented edition, Walter de Gruyter, Berlin, 2013.

[9] J. F. DAvis AND P. KIRK, "Lecture Notes in Algebraic Topology", Graduate Studies in Mathematics 35, American Mathematical Society, Providence, RI, 2001. DOI: 10.1090/gsm/035.

[10] G. DE RhAm, Introduction aux polynômes d'un nœud, Enseignement Math. (2) 13 (1967), 187-194 (1968).

[11] C. McA. Gordon, Some aspects of classical knot theory, in: "Knot Theory" (Proc. Sem., Plans-sur-Bex, 1977), Lecture Notes in Math. 685, Springer, Berlin, 1978, pp. 1-60.

[12] M. Heusener and O. Medjerab, Deformations of reducible representations of knot groups into $\mathrm{SL}(n, \mathbf{C})$, Math. Slovaca 66(5) (2017), 1337-2211. DOI: 10.1515/ms-2016-0206.

[13] M. Heusener And J. Porti, Deformations of reducible representations of 3-manifold groups into $\mathrm{PSL}_{2}(\mathbb{C})$, Algebr. Geom. Topol. 5 (2005), 965-997. DOI: 10.2140/agt.2005.5.965.

[14] M. Heusener, J. Porti, And E. SuÁrez Peiró, Deformations of reducible representations of 3-manifold groups into $\mathrm{SL}_{2}(\mathbb{C})$, J. Reine Angew. Math. 530 (2001), 191-227. DOI: 10.1515/crll.2001.003.

[15] W. JACO, "Lectures on Three-Manifold Topology", CBMS Regional Conference Series in Mathematics 43, American Mathematical Society, Providence, R.I., 1980.

[16] H. Jebali, Module d'Alexander et représentations métabéliennes, Ann. Fac. Sci. Toulouse Math. (6) 17(4) (2008), 751-764. DOI: 10.5802/afst. 1201. 
[17] A. Lubotzky and A. R. Magid, Varieties of representations of finitely generated groups, Mem. Amer. Math. Soc. 58(336) (1985), 117 pp. DOI : $10.1090 / \mathrm{memo} / 0336$.

[18] W. A. Stein ET AL., Sage Mathematics Software (Version 6.9), The Sage Development Team (2015). http://www. sagemath.org.

[19] A. WeIL, Remarks on the cohomology of groups, Ann. of Math. (2) 80(1) (1964), 149-157. DOI: 10.2307/1970495.

Leila Ben Abdelghani:

Faculté des Sciences de Monastir

Boulevard de l'environnement

5019 Monastir

Tunisie

E-mail address: leila.benabdelghani@gmail.com

Michael Heusener:

Université Clermont Auvergne

CNRS

Laboratoire de Mathématiques Blaise Pascal

F-63000 Clermont-Ferrand

France

E-mail address: michael.heusener@uca.fr

Primera versió rebuda el 24 d'agost de 2015, darrera versió rebuda el 13 d'abril de 2016. 